WATER LEVELS IN NASSAU COUNTY SEWER DISTRICTS 2 AND 3,

LONG ISLAND, NEW YORK, 1978-79

By Henry F. H. Ku and Dale L. Simmons

Water-Resources Investigations

Report 82-4017

Prepared in cooperation with

NASSAU COUNTY DEPARTMENT OF PUBLIC WORKS

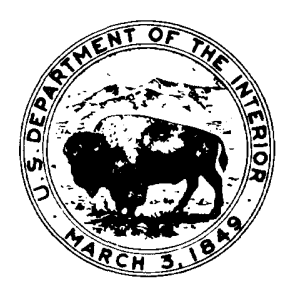

Syosset, New York 
UNITED STATES DEPARTMENT OF THE INTERIOR

JAMES G. WATT, Secretary

GEOLOGICAL SURVEY

Dallas L. Peck, Director

For additional information write to:

U. S. Geological Survey

5 Aerial Way

Syosset, New York 11791
Copies of this report may be purchased from:

U.S. Geological Survey Western Distribution Branch Open-File Services Section Box 25425, Denver Federal Center Denver, Colo. 80225 


\section{CONTENTS}

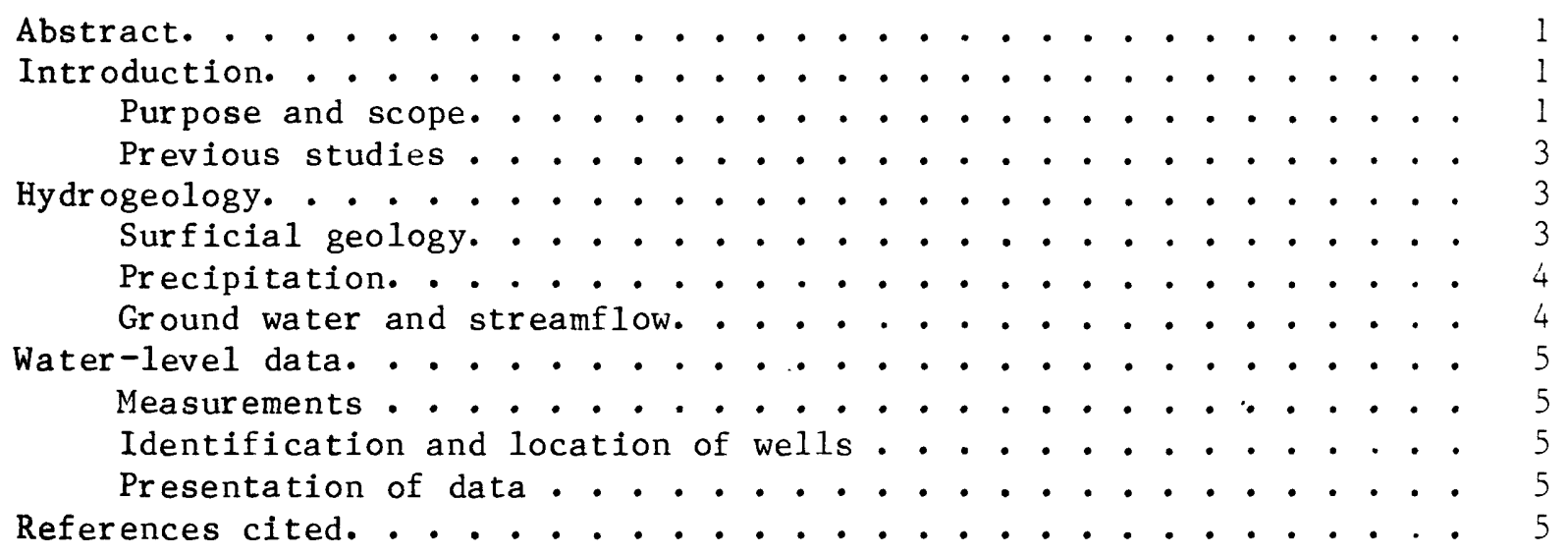

\section{ILLUSTRATION}

Figure 1. Map showing location and principal hydrologic features of area studied, Nassau County, N. Y. . . . . . . . . . .

\section{TABLES}

Table 1. Generalized description of hydrogeologic units underlying study area. . . . . . . . . . . . . . . . . . 4

2. Water levels in wells in Nassau County Sewer Districts

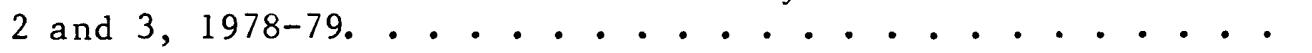

\section{PLATE \\ (in pocket)}

Plate 1. Map showing location of measured wells, Nassau County Sewer Districts 2 and 3. 


\section{CONVERSION FACTORS AND ABBREVIATIONS}

The following factors may be used to convert units used in this report to the International System (SI) of Units.

Multiply Inch-pound unit

$\underline{B y}$

foot $(f t)$

0.3048

mile (mi)

1.609

inch (in)

25.4

feet per mile ( $\mathrm{ft} / \mathrm{mi}$ )

0.1894

\section{To obtain SI unit}

meter (m)

kilometer ( $\mathrm{km})$

millimeter (mm)

meter per kilometer

$(\mathrm{m} / \mathrm{km})$ 


\title{
WATER LEVELS IN NASSAU COUNTY SEWER DISTRICTS 2 AND 3, LONG ISLAND, NEW YORK, 1978-79
}

\author{
By
}

Henry F. H. Ku and Dale L. Simmons

\begin{abstract}
Prior to development on Long Island, about 95 percent of total streamflow originated as ground-water seepage from the upper glacial aquifer, the remaining 5 percent consisted of direct storm runoff. Thus, the streams of Long Island function as ground-water drains. Their flow in dry weather is directly controlled by ground-water levels adjacent to the stream channels.

Sewerage systems in Nassau County have caused a general lowering of ground-water levels, which in turn has severely reduced streamflow. This report presents monthly water-level measurements in 259 wells in Nassau County from 1978-79 to provide a data base to evaluate the need to replace streamflow lost through lowering of the water table.
\end{abstract}

\section{INTRODUCTION}

An extensive sanitary sewer network has been constructed in western Nassau County in an attempt to improve ground-water quality by intercepting wastewater that would otherwise contaminate the ground-water reservoir. This practice has resulted in a loss of recharge to the upper glacial aquifer with a consequent lowering of the water table.

Because the base flow of Long Island's streams during dry weather is directly dependent on ground-water levels in the area adjacent to the streams, increased ground-water withdrawals in Sewer District 2 and the construction of additional sanitary sewers in Sewer District 3 have raised questions as to the anticipated magnitude of reduction in streamflow. The locations of the sewer districts, the streams, and the regional ground-water divide in the study area are shown in figure 1. Several measures have been proposed to mitigate the effects of sewerage on streamflow; their effectiveness will be evaluated by a digital computer model of Long Island's hydrologic system.

\section{Purpose and Scope}

This report presents a list of ground-water levels in 259 wells in Nassau County Sewer Districts 2 and 3 from 1978-79. The data were collected as a part of work done in cooperation with Nassau County Department of Public Works in response to grant condition 3 of the U.S. Environmental Protection Agency project C36-982.08. The, purpose of the study was to collect detailed waterlevel information needed for calibration of the U.S. Geological Survey's 


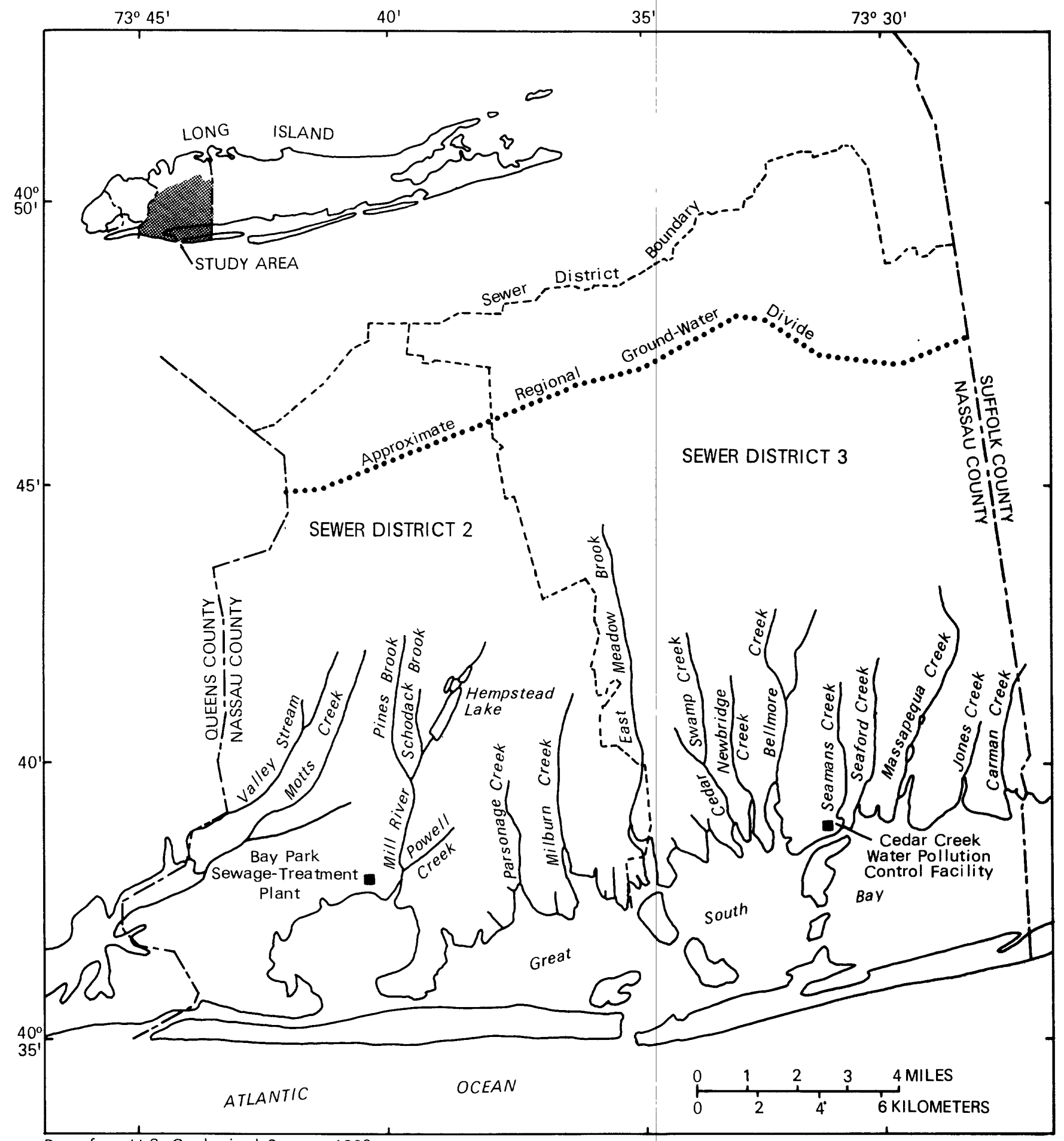

Base from U.S. Geological Survey, 1980

State base map, 1974

Figure 1.--Location and principal hydrologic features of area studied, Nassau County, N.Y. (Modified from Ku and Simmons, 1981.) 
digital computer model of ground-water flow. The model will be used to predict the effects of selected short-term actions proposed to offset the expected decrease in ground-water levels and to investigate other changes that may result from the increased use of the Bay Park Sewage Treatment Plant in Sewer District 2 and construction of new sanitary sewers in Sewer District 3.

\section{Previous Studies}

A report by $\mathrm{Ku}$ and Simmons (1981) presents data on the base flow of streams in the area studied. The base-flow data in that report were collected simultaneously with the water-level information presented herein.

Related studies concerning analog-model analysis of the effects of sewerage on ground-water levels are described by Kimmel and Harbaugh (1976) and $\mathrm{Ku}$ and Sulam (1979). Garber and Sulam (1976), Pluhowski and Spinello (1978), and Sulam (1979) also discussed the relationship between declining water levels and sewerage. Reynolds (1982) and Simmons and Reynolds (1982) discussed the effects of the lowered water table on stream base flow. A recent map of the water table on Long Island is included in Donaldson and Koszalka (1982).

\section{HYDROGEOLOGY}

The geology and hydrology of Long Island have been summarized in many reports. Cohen and others (1968) gave a general overview of the hydrology of Long Island, and Perlmutter and Geraghty (1963) presented a detailed analysis of the geology and ground-water conditions in southern Nassau County.

\section{Surficial Geology}

Surficial deposits on Long Island are the result of two separate advances of the Wisconsinan glacier. Two terminal moraines form east-west-trending lines of hills that reach a maximum altitude of about 400 feet. These moraines, which consist of poorly sorted debris including boulders, gravel, sand, silt, and clay, merge in the western part of the island. A gently sloping outwash plain composed of well-sorted glaciofluvial sand and gravel extends southward from the line of moraines to the south shore with a slope of about 20 feet per mile (Cohen and others, 1968).

The high permeability of the glacial outwash sand and gravel, in addition to the flat terrain, cause most precipitation to infiltrate into the ground almost immediately. Under natural (predevelopment) conditions, about 95 percent of total streamflow originates as ground-water seepage from the upper glacial aquifer (Franke and McClymonds, 1972); the remaining 5 percent consists of direct runoff. Thus, the streams of Long Island function as ground-water drains. In dry weather their flow is directly controlled by ground-water levels adjacent to the stream channels (Pluhowski and Kantrowitz, $1964)$. 


\section{Precipitation}

Mean annual precipitation on Long Island ranges from slightly less than 41 inches on the south shore to slightly more than 50 inches in the island's central region (Miller and Frederick, 1969); the long-term mean is 44 inches (Cohen and others, 1968). Average warm-season and cool-season precipitation totals are almost equal.

\section{Ground Water and Streamflow}

The ground-water system of Long Island consists of four major aquifers (table 1). The lower three are confined (artesian); the water-table (upper glacial) aquifer is hydraulically connected to the streams and lakes of the island. The water-table aquifer is the source of base flow for all the island's streams but is no longer extensively used for public water supply in Nassau County because it is contaminated from sources such as septic-tank and cesspool discharges and fertilizers.

Table 1.--Generalized description of hydrogeologic units underlying study area. ${ }^{1}$

Hydrogeologic Unit

Lithology and water-bearing character

Upper glacial aquifer

Gardiners clay

Jameco aquifer

Magothy aquifer

Raritan confining unit

Lloyd aquifer
Mainly sand and gravel of moderate to high permeability; also includes clayey till of low permeability.

Clay, silty clay, and a little fine sand of low to very low permeability.

Mainly medium to coarse sand of moderate to high permeability.

Coarse to fine sand of moderate permeability; locally contains gravel of high permeability and abundant silt and clay of low to very low permeability.

Clay of very low permeability; some silt and fine sand of low permeability.

Sand and gravel of moderate permeability; some clayey material of low permeability.

Modified from Cohen and others (1968). 
In general, water levels on most of Long Island rose during the early 1970 's as a result of above-average precipitation in 1972-73 (Koszalka, 1975). During the same period, however, water levels in eastern Queens and southwestern Nassau County declined 3 to 4 feet. Koszalka (1975, p. 4) attributes this decline to the increase in local ground-water development and to sanitary sewerage in southwestern Nassau County. The water-table configuration has changed little since 1975 except for rises of 5 feet in central Nassau County and 9 feet in central Suffolk County (Donaldson and Koszalka, 1982).

\section{WATER-LEVEL DATA}

\section{Measurements}

Water-level measurements were made monthly from April 1978 to May 1979 in 259 wells in Nassau County Sewer Districts 2 and 3 . Well locations are shown on plate 1. Each month the wells were measured during a 2- to 3-day period after several days without precipitation to coincide with the concurrent base-flow measurements made in nearby streams ( $\mathrm{Ku}$ and Simmons, 1981).

\section{Identification and Location of Wells}

All wells measured during this stucy are in Nassau County. Hence, all well numbers in table 2 and plate 1 are preceded by the letter "N." Well numbers are those assigned by the New York State Department of Environmental Conservation.

\section{Presentation of Data}

Table 2 (at end of report) presents the water-level data collected during this study (April 1978 - May 1979). Wells are listed in numerical order, followed by latitude and longitude. Measurement dates and water levels, in feet above sea level, are given for each well. (Sea level refers to National Geodetic Vertical Datum of 1929.)

All original data are available for inspection at the U.S. Geological Survey office, 5 Aerial Way, Syosset, N. Y. 11791.

\section{REFERENCES CITED}

Cohen, Philip, Franke, 0. L., and Foxworthy, B. L., 1968, An atlas of Long Island's water resources: New York State Water Resources Commission Bulletin 62, $117 \mathrm{p}$.

Donaldson, C. D. , and Koszalka, E. J., 1982, The water table on Long Island, New York, March 1979: U.S. Geological Survey Open-File Report 82-162, 2 sheets. 


\section{REFERENCES CITED (continued)}

Franke, O. L., and McClymonds, N. E., 1972, Summary of the hydrologic situation on Long Island, New York, as a guide to water-management alternatives: U.S. Geological Survey Proflessional Paper 627-F, 59 p.

Garber, M. S., and Sulam, D. J., 1976, Factors affecting declining water levels in a sewered area of Nassau County, New York: U.S. Geological Survey Journal of Research, v. 4, no. 3, p. 255-265.

Kimme1, G. E., and Harbaugh, A. W., 1976, Analog-model analysis of effects of waste-water management on the ground-water reservoir in Nassau and Suffolk Counties, New York, Report 1--Proposed and current sewerage: U.S. Geological Survey Open-File Report 76-441, 32 p.

Koszalka, E. J., 1975, The water table on Long Island, New York, in March 1974: Long Island Water Resources Bulletin 5, 7 p.

$\mathrm{Ku}$, H. F. H., and Simmons, D. L., 1981, Base flow of streams in Nassau County Sewer Districts 2 and 3, Long Island, New York, 1978-79: U.S. Geological Survey Water-Resources Investigations, Open-File Report $81-420,32 \mathrm{p}$.

Ku, H. F. H., and Sulam, D. J., 1979, Hydrologic and water-quality appraisal of southeast Nassau County, Long Island, New York: Long Island Water Resources Bulletin 13, 129 p.

Miller, J. F., and Frederick, R. H., 1969, The precipitation regime of Long Island, New York: U.S. Geological Survey Professional Paper 627-A, 21 p.

Per lmutter, N. M. , and Geraghty, J. J., 1963, Geology and ground-water conditions in southern Nassau and southeastern Queens Counties, Long lsland, New York: U.S. Geological Survey Water-Supply Paper 1613-A, 205 p.

Pluhowski, E. J., and Kantrowitz, I. H., 1964, Hydrology of the Babylon-lslip area, Suffolk County, Long Island, New York: U.S. Geological Survey Water-Supply Paper 1768, 119 p.

Pluhowski, E. J., and Spinello, A. G., 1978, Impact of sewerage systems on stream base flow and ground-water recharge on Long Island, New York: U.S. Geological Survey Journal of Research, v. 6, no. 2, p. 263-271.

Reynolds, R. J., 1982, Base flow of Long Island streams, 1960-75: U.S. Geological Survey Water-Resources Investigations 81-48, 33 p. 


\section{REFERENCES CITED (continued)}

Simmons, D. L., and Reynolds, R. J., 1982, Effects of urbanization on base flow of selected south-shore streams, Long Island, New York: Water Resources Bulletin, v. 18, no. 5, p. 797-805.

Sulam D. J., 1979, Analysis of changes in ground-water levels in a sewered and an unsewered area of Nassau County, Long Island, New York: Ground Water, v. 17 , no. 5, p. 446-455. 


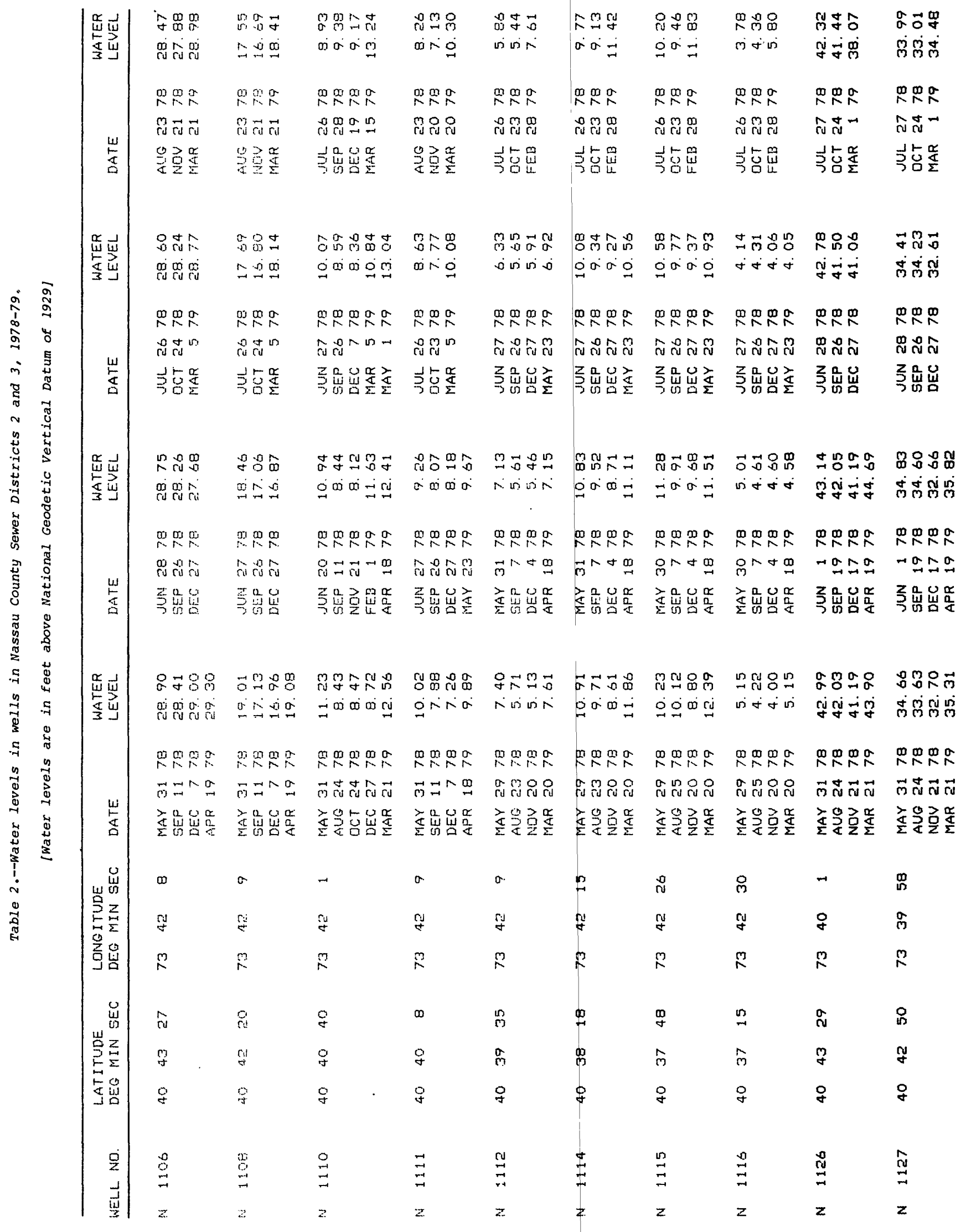




\begin{tabular}{|c|c|c|c|c|c|c|c|c|c|c|}
\hline 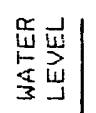 & 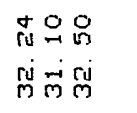 & 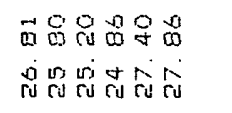 & 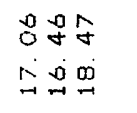 & $\begin{array}{l}\hat{0}+5 \\
00=\end{array}$ & 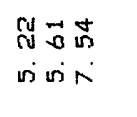 & 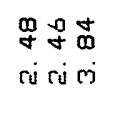 & 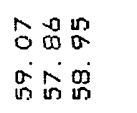 & 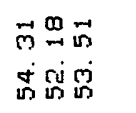 & 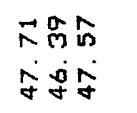 & $\begin{array}{l}\overrightarrow{0} 0 \text { 品 } \\
\text { 땅워 }\end{array}$ \\
\hline & $\stackrel{\text { ma }}{\wedge}$ & 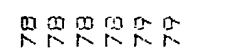 & $\stackrel{m}{\wedge}$ & $\stackrel{p}{N} \mathbb{R}$ & $\stackrel{p o n}{R}$ & 果是 & $\stackrel{m}{\wedge}$ & mpgra & $\stackrel{m}{\wedge} \stackrel{\infty}{\wedge}$ & $\stackrel{\infty}{\wedge} \underset{\wedge}{\infty}$ \\
\hline & रिं त्र & 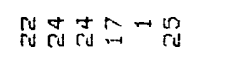 & ति 品 & 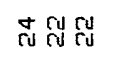 & 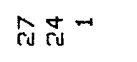 & तิ स्षे & กึ लु & तก กู & 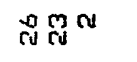 & กู \\
\hline 峁 & $\begin{array}{l}3 \\
3\end{array}$ & 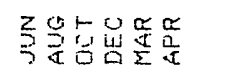 & 宂它采 & 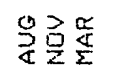 & 引ち皆京 & 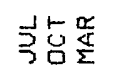 & 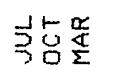 & 引皆变 & 引ち造充 & 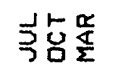 \\
\hline 号岀 & 恖息品 & 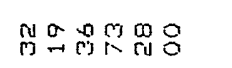 & $\stackrel{\text { man }}{\wedge}$ & 点品品 & 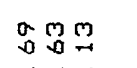 & 星战 & 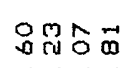 & 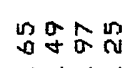 & 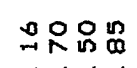 & 뭇욤 \\
\hline 虽出 & लिं & ru & $=\stackrel{D}{\square}$ & $00 \pm$ & in in & ci ri ri & 舟量的品 & 的舀品品 & ஷ் & 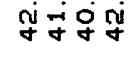 \\
\hline & $\stackrel{p}{R} \stackrel{p}{R}$ & 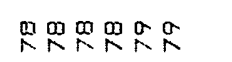 & 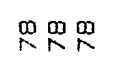 & $\stackrel{p}{p} \underset{\wedge}{\infty}$ & $\stackrel{\infty}{\wedge} \stackrel{m}{\wedge}$ & 象只只 & $\stackrel{m}{\wedge} \stackrel{0}{\wedge} \underset{\wedge}{\wedge}$ & 只果只只 & 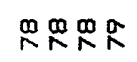 & $m^{m} \mathbb{m}^{\infty} \stackrel{\alpha}{\wedge}$ \\
\hline & 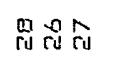 & 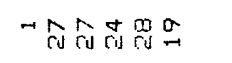 & 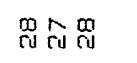 & त̂ & ำ & 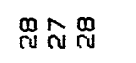 & ㅅำ & 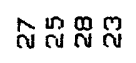 & ล & 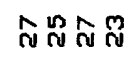 \\
\hline$\frac{\underline{W}}{\underline{\sigma}}$ & 㕺岕点 & 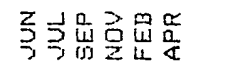 & $\sum_{3} \frac{n}{w}$ & 引ち选 & 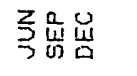 & 勇出㟧 & 强㟧衣 & 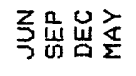 & 弓品㟧充 & 枵品出这 \\
\hline 觅岁 & 酷合品芯 & กํํำ & 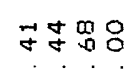 & 미 몽 & 800 & ำํำ & $\stackrel{\infty}{\wedge}$ 웜웜 & 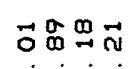 & 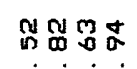 & \pm 웅 \\
\hline$\frac{2}{3}$ & लिं लें & 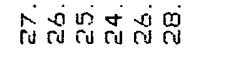 & $\stackrel{m}{\rightarrow}=0$ & 000 & 0 in is & mंर्च m & 舟嵒的品 & 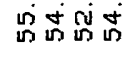 & 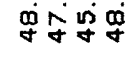 & 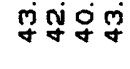 \\
\hline & $\stackrel{m}{\wedge} \stackrel{\infty}{\wedge} \stackrel{\infty}{\wedge} \stackrel{\sigma}{\wedge}$ & MRRRRR & 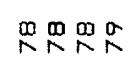 & $\stackrel{m}{\wedge} \stackrel{m}{\wedge}$ & mpgr & mon & $\stackrel{m}{\wedge} \mathbb{R} R \mathbb{R}$ & 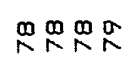 & 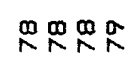 & 番果界员 \\
\hline & $-\sigma=\sigma$ & 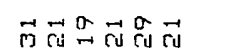 & $\rightarrow \sigma=0$ & 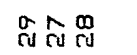 & $+\infty-1$ & $-\infty+a$ & 凹๐י & 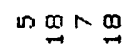 & 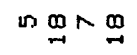 & $\ddot{m} \sim \mathscr{m}$ \\
\hline$\stackrel{5}{5}$ & 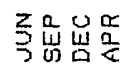 & 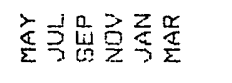 & 至出岩哭 & 引品㟧 & 引品范 & 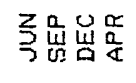 & 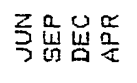 & 引品岩哭 & 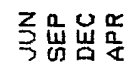 & 引品㟧器 \\
\hline 要要 & Nog品 & 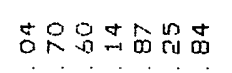 & 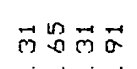 & 药哭 & 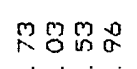 & ㅅํำ กิ & 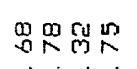 & 品合觉只 & MNำ & 옹용 \\
\hline 莩 & में लंखें & 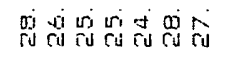 & 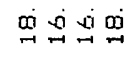 & $\circ \sigma 00$ & sin is ris & மं ri ru mj & 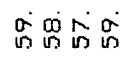 & 落的落 & 乎安毞安 & 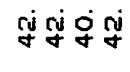 \\
\hline & $\stackrel{\infty}{\wedge} \stackrel{\infty}{\wedge} \stackrel{\infty}{\wedge}$ & 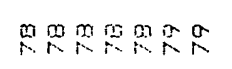 & 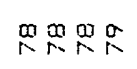 & 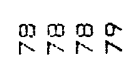 & 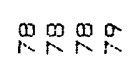 & 番界界是 & magn & 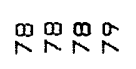 & 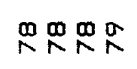 & 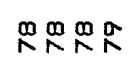 \\
\hline & ले ru ru ru & 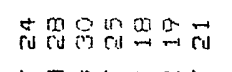 & 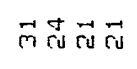 & 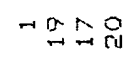 & 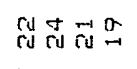 & 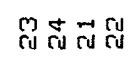 & 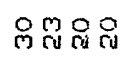 & 윰ำ & 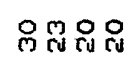 & 윰ำ \\
\hline 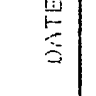 & 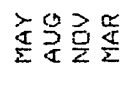 & 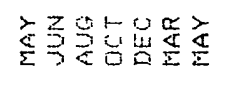 & 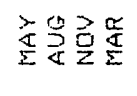 & 强品㟔品 & 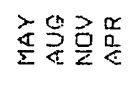 & 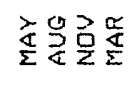 & 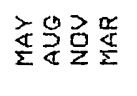 & 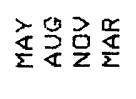 & 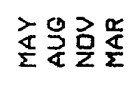 & 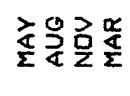 \\
\hline 岁 & in & $\stackrel{\mathfrak{g}}{q}$ & $\stackrel{\mathfrak{m}}{\mathfrak{B}}$ & $\stackrel{\sigma}{m}$ & m & ले & 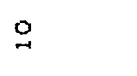 & + & $\mathfrak{N}$ & $\stackrel{0}{+}$ \\
\hline$=$ & 命 & $\stackrel{j}{p}$ & a & $\hat{m}$ & m & 㖣 & 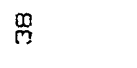 & $\underset{m}{\mathfrak{m}}$ & $\hat{m}$ & $\hat{\mathfrak{m}}$ \\
\hline 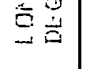 & ? & $R$ & $\stackrel{m}{R}$ & $\stackrel{m}{R}$ & $\stackrel{M}{R}$ & $\stackrel{M}{R}$ & ? & $\stackrel{m}{\wedge}$ & 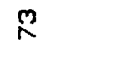 & $\stackrel{3}{\wedge}$ \\
\hline 菢 & M & ri & $\vec{\sigma}$ & ba & ry & $\stackrel{n}{m}$ & $\vec{\sigma}$ & $\vec{q}$ & + & ते \\
\hline 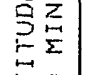 & $\stackrel{\text { gy }}{q}$ & $\vec{\sigma}$ & $\stackrel{9}{q}$ & $\stackrel{\circ}{q}$ & $\stackrel{a}{a}$ & $\stackrel{m}{m}$ & g & $\stackrel{9}{9}$ & gु & y \\
\hline 点 & 웅 & o & 웜 & $\stackrel{9}{4}$ & q & 웅 & 워 & 운 & 웅 & 웜 \\
\hline 운 & 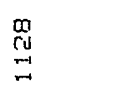 & 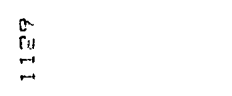 & $\begin{array}{l}0 \\
\stackrel{8}{7} \\
=\end{array}$ & $\stackrel{\overrightarrow{0}}{\Xi}$ & $\underset{\substack{y \\
0 \\
-7}}{-1}$ & $\stackrel{9}{9}$ & $\begin{array}{c}0 \\
0 \\
07 \\
-7\end{array}$ & $\underset{q}{q}$ & $\stackrel{\vec{J}}{\vec{H}}$ & $\stackrel{\text { \} }{\beth}} \\
{\hline \text { 荘 }} &{z} &{z} &{=.} &{z} &{z} &{z} &{z} &{z} &{z} &{z} \\
$\hline
\end{tabular}




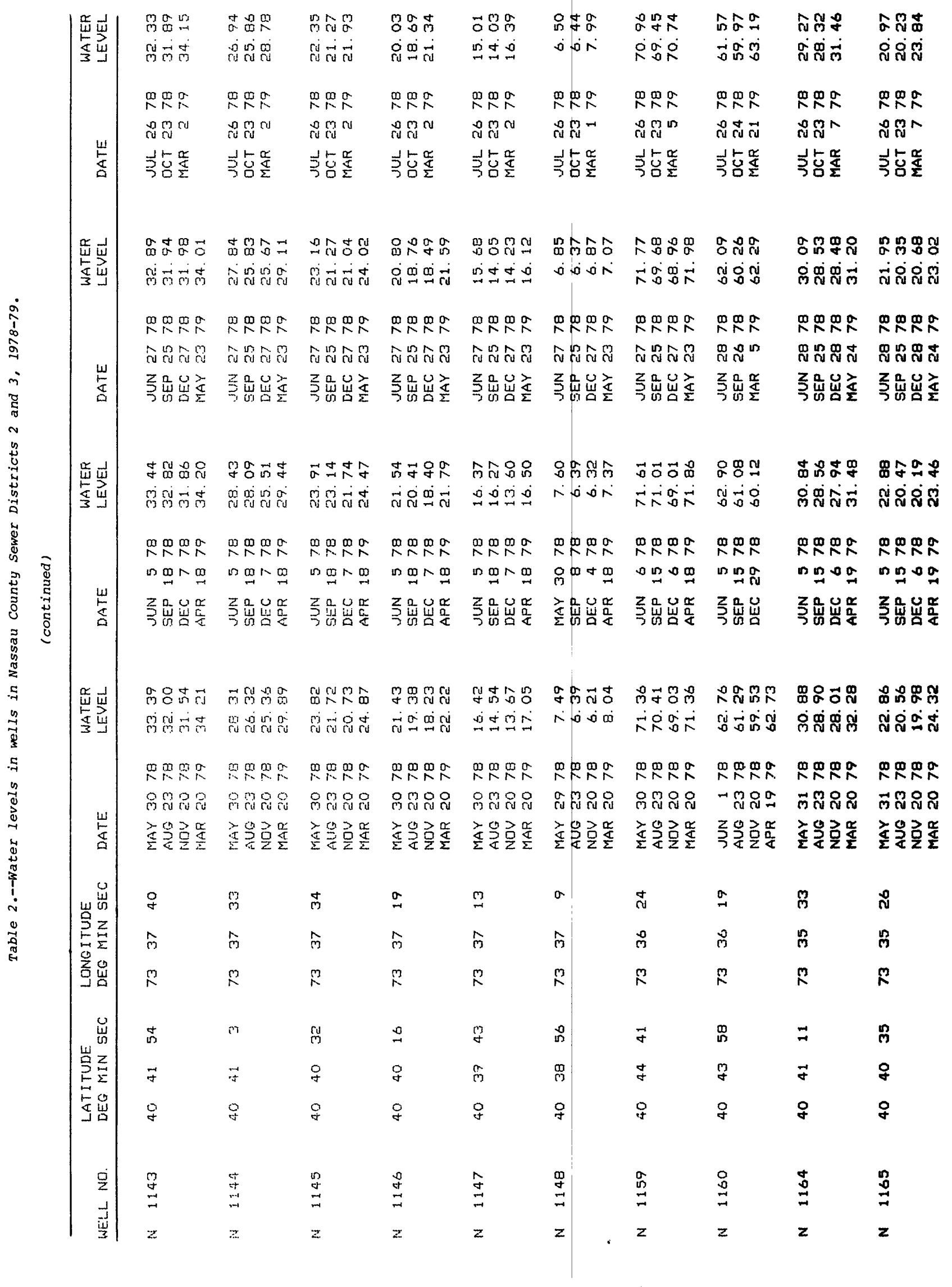




\begin{tabular}{|c|c|c|c|c|c|c|c|c|c|c|c|}
\hline 妥岕 & $\begin{array}{l}0=0 \\
0=1 \\
\dot{\square} \dot{\square} 0\end{array}$ & 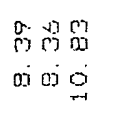 & 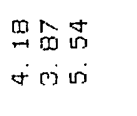 & 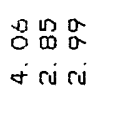 & 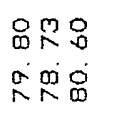 & 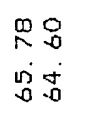 & 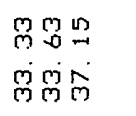 & 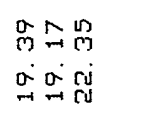 & $\begin{array}{l}9 M= \\
097 \\
+\square 0\end{array}$ & 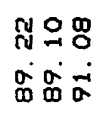 & 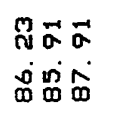 \\
\hline $\begin{array}{l}\stackrel{\omega}{\mathrm{E}} \\
\stackrel{\Delta}{\Delta}\end{array}$ & 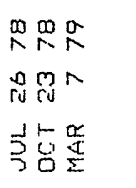 & 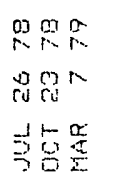 & 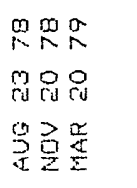 & 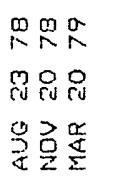 & 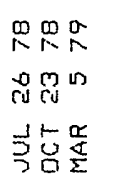 & 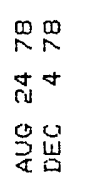 & 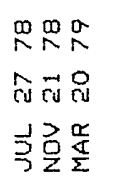 & 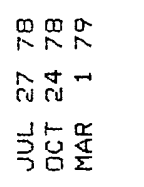 & 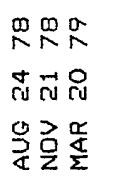 & 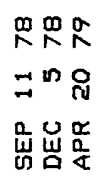 & 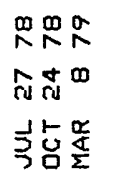 \\
\hline 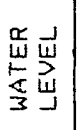 & 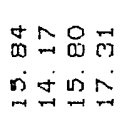 & 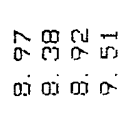 & 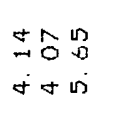 & 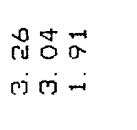 & 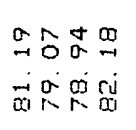 & 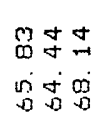 & 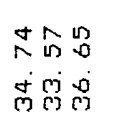 & 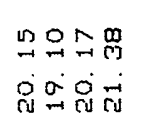 & 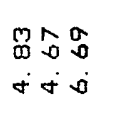 & 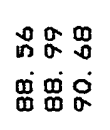 & 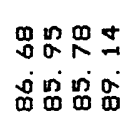 \\
\hline 峁 & 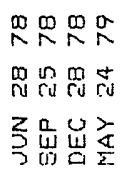 & 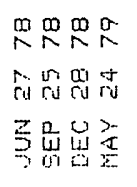 & 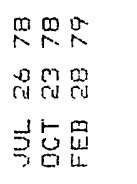 & 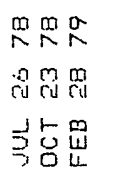 & 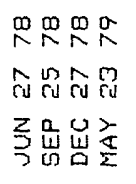 & 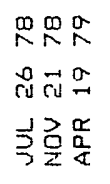 & 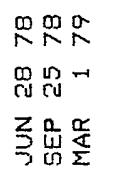 & 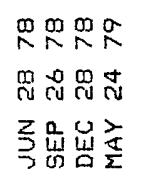 & 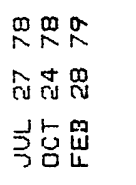 & 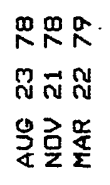 & 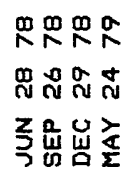 \\
\hline 足岃 & 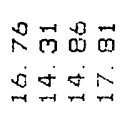 & 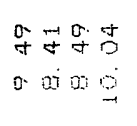 & 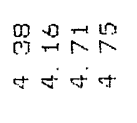 & 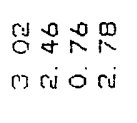 & 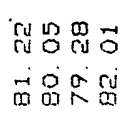 & 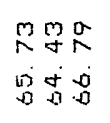 & 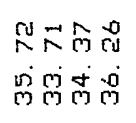 & 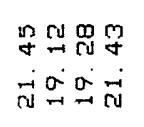 & 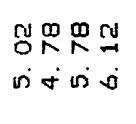 & 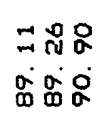 & 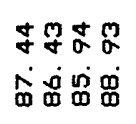 \\
\hline 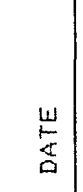 & 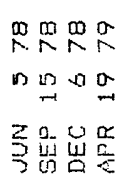 & 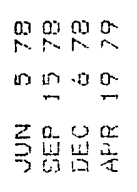 & 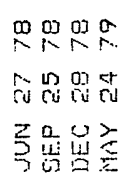 & 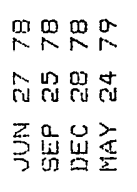 & 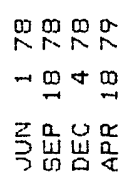 & 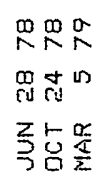 & 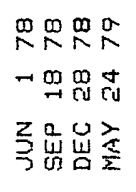 & 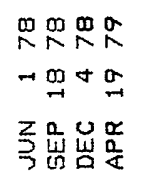 & 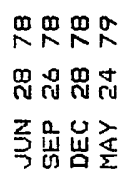 & 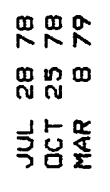 & 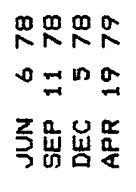 \\
\hline 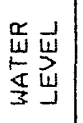 & 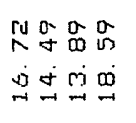 & 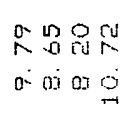 & 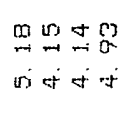 & 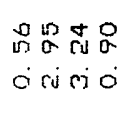 & 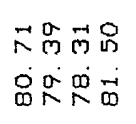 & $\begin{array}{l}\hat{n} 9 \\
\text { ngigig } \\
\text { prog }\end{array}$ & 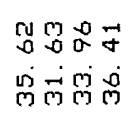 & 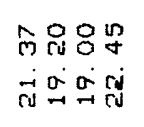 & 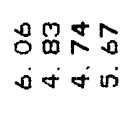 & 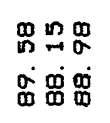 & 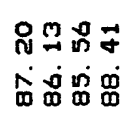 \\
\hline $\begin{array}{l}\omega \\
\stackrel{L}{a} \\
\end{array}$ & 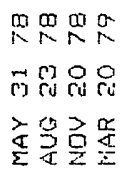 & 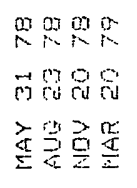 & 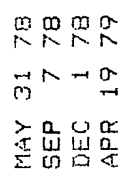 & 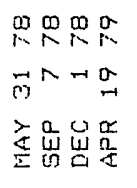 & 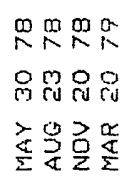 & 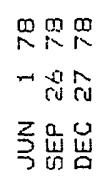 & 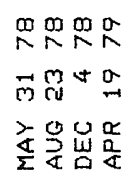 & 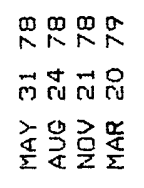 & 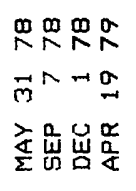 & 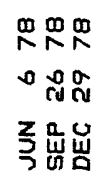 & 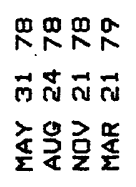 \\
\hline 㟧 & $\stackrel{n d v}{v}$ & \pm & $M$ & $\rightarrow$ & 怘 & ตे & $\wedge$ & 品 & $\underset{\sim}{\mathbb{N}}$ & $\exists$ & $m$ \\
\hline 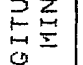 & $\stackrel{n}{7}$ & 品 & 9 & $\vec{m}$ & ले & 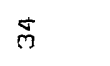 & ले & ल & M & ஜำ & Пี \\
\hline 总总 & $R^{2}$ & $\stackrel{M}{R}$ & $\stackrel{M}{n}$ & $M^{M}$ & $\stackrel{R}{R}$ & $\stackrel{\mathfrak{M}}{\wedge}$ & $\stackrel{m}{\wedge}$ & $\stackrel{M}{R}$ & $\stackrel{M}{\wedge}$ & $\stackrel{m}{R}$ & $\stackrel{n}{\wedge}$ \\
\hline 岀 & - & 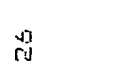 & $\stackrel{⿰ 习 习}{\dot{q}}$ & 0 & $\stackrel{\sigma}{a}$ & $\Rightarrow$ & $\stackrel{0}{m}$ & $\hat{m}$ & $\underset{-1}{M}$ & ru & $\sigma$ \\
\hline 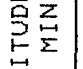 & 움 & 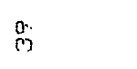 & 羿 & 品 & $\stackrel{n g}{q}$ & $\underset{f}{+}$ & $\vec{q}$ & g & $\stackrel{\sigma}{m}$ & $\hat{\sigma}$ & +̊ \\
\hline 廷总 & \& & 品 & 앙 & $q$ & 암 & 움 & $q$ & 움 & 웅 & 웅 & 웅 \\
\hline$\frac{9}{2}$ & 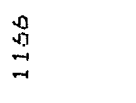 & 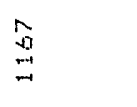 & $\begin{array}{l}0 \\
0 \\
0 \\
=-1 \\
=\end{array}$ & $\begin{array}{c}a \\
\substack{0 \\
n \\
n-1} \\
-1\end{array}$ & $\stackrel{\infty}{\stackrel{\infty}{=}}$ & $\begin{array}{l}0 \\
9 \\
\underset{-1}{-1}\end{array}$ & \begin{tabular}{l}
$\stackrel{9}{0}$ \\
$\stackrel{0}{\Xi}$ \\
\hdashline
\end{tabular} & $\underset{⿱ 中}{ \pm}$ & 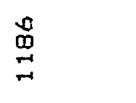 & $\stackrel{+}{\sigma}$ & $\stackrel{n}{a}$ \\
\hline$\frac{4}{3}$ & $z$ & $z$ & $z$ & $z$ & $z$ & $z$ & $z$ & $z$ & $z$ & $z$ & $z$ \\
\hline
\end{tabular}




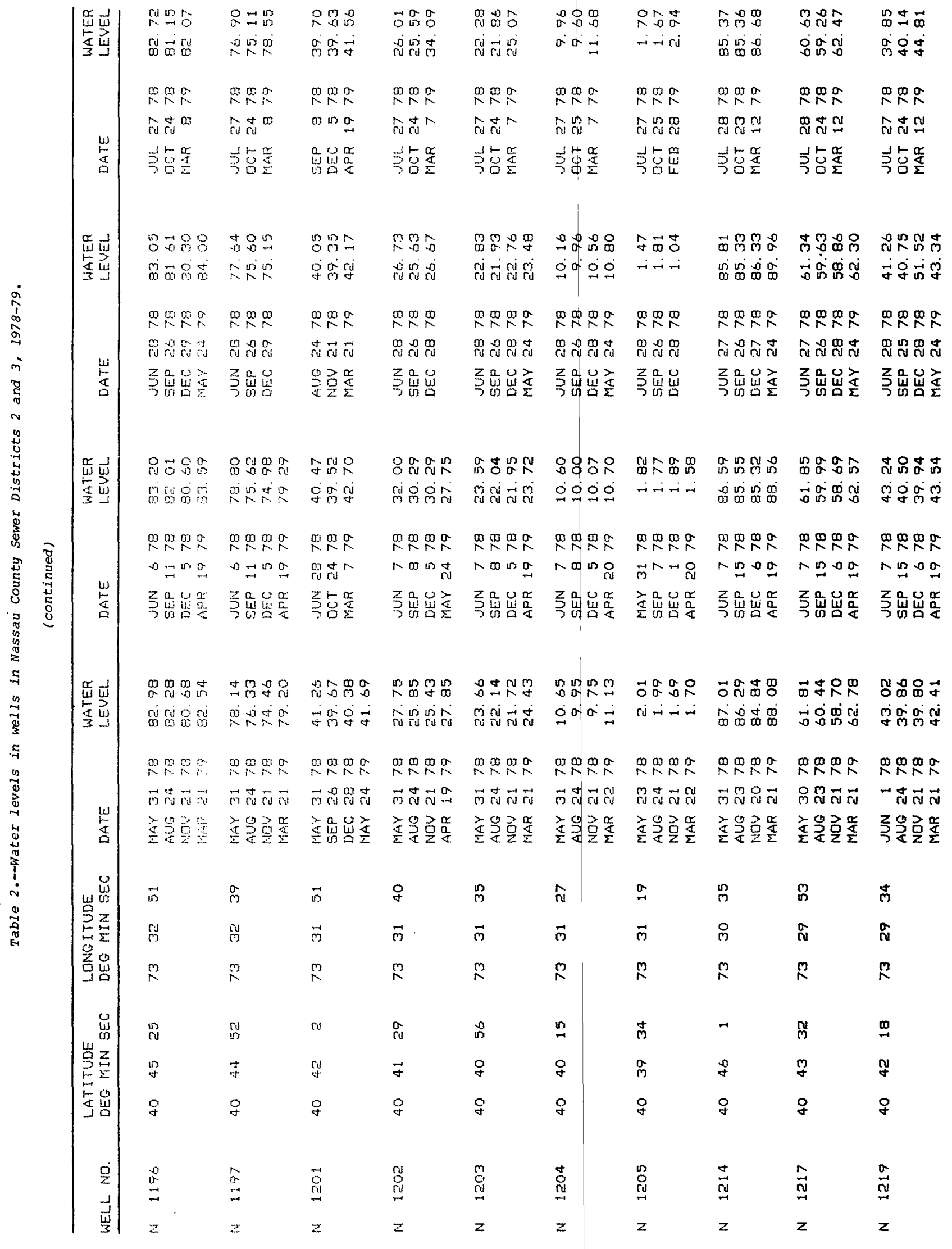




\begin{tabular}{|c|c|c|c|c|c|c|c|c|c|c|}
\hline \multirow{2}{*}{ 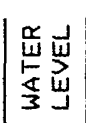 } & 맴용 & 乎品哭 & 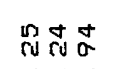 & rung & กิ่ & 哭品字 & 路品 & $\hat{\infty}=\mathbf{n}$ & 品品合 & $\sigma \sin$ \\
\hline & 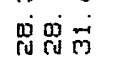 & $a \sigma r j$ & o0ं & ri rirj & म्: & 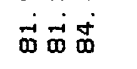 & 0 & 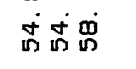 & in & ติต \\
\hline & $\stackrel{p}{R} \stackrel{p}{R} R$ & 果界是 & 只照是 & 骂界㫔 & $\stackrel{p a r}{\wedge} \stackrel{0}{\wedge}$ & 界界六 & $\stackrel{\infty}{\wedge} \underset{\wedge}{R} \sigma$ & $\stackrel{R}{N} \stackrel{0}{\wedge}$ & 象界昂 & 界界是 \\
\hline & 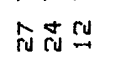 & 合苋等 & 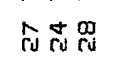 & 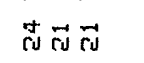 & 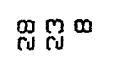 & 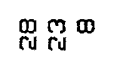 & กำ & $\underset{\text { cos }}{\operatorname{mov}} \wedge$ & กำ & ก็ณู \\
\hline 点 & $35 \frac{\alpha}{3}$ & 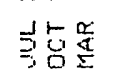 & 35면 & 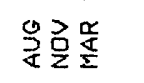 & 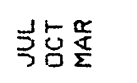 & $35 \frac{x}{5}$ & 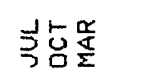 & 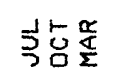 & jち皆 & 引吉出㐫 \\
\hline 品 & 员员合 & O゚马士 & 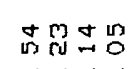 & 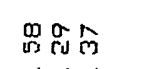 & 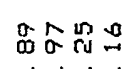 & 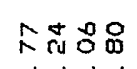 & 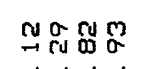 & ํํำํํำ & gू & 무중요 \\
\hline 总 & 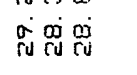 & QDo & a.0잉 & ri rif & 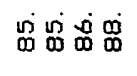 & 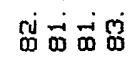 & 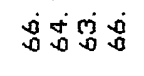 & 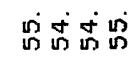 & 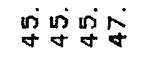 & ติต \\
\hline & 秥只品 & 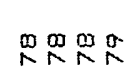 & mon & pqRa & 品品品只 & 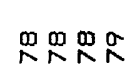 & 䎹象只 & פำ & $\stackrel{m}{\wedge} \stackrel{0}{\wedge} \stackrel{0}{\wedge}$ & 果是界只 \\
\hline & 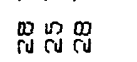 & 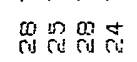 & 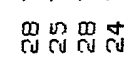 & 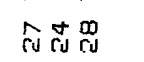 & 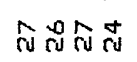 & 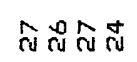 & 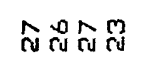 & 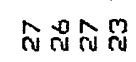 & 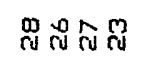 & กิณี \\
\hline 崖 & 至品㟧 & 强出委 & 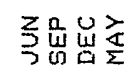 & 方品出 & 强苫㟧交 & 亭品㟧文 & 强㟧岩变 & 号品崖文 & 强品㟧充 & 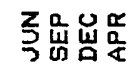 \\
\hline 品 & กำ & 的品跑品 & 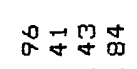 & 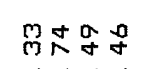 & 몽워소쇼 & 红品员品 & 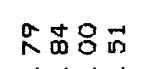 & 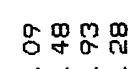 & 界命员 & 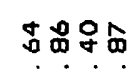 \\
\hline 年岁 & 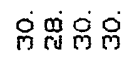 & ciug & $\sigma \sigma \sigma \sigma$ & ri ri ri mi & 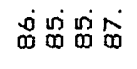 & 迁官品 & 0 & 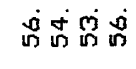 & $\dot{q}+\dot{q} \dot{q} \dot{q}$ & ติ ตूं \\
\hline & 舟界界是 & 塄罢界只 & mag & 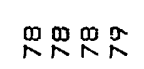 & 只只只只 & 采只品员 & 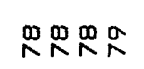 & 舟象员员 & 只塄只员 & 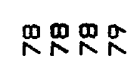 \\
\hline & $\therefore \approx 0 \sigma$ & $\operatorname{ng} 00$ & $\rightarrow n-\sigma$ & 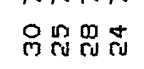 & $\ln _{\rightarrow}$ & $\operatorname{ngn} \theta$ & $\ln _{n} \mathbb{\sigma}$ & $\sin \theta$ & $\Lambda \underset{T}{\operatorname{mos}}$ & 믐요 \\
\hline 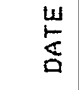 & 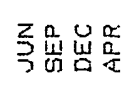 & 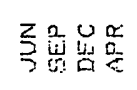 & 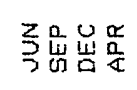 & 引品岩充 & 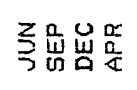 & 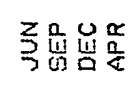 & 弓品㟔哭 & ว品出器 & 强岩哭 & 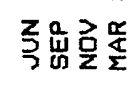 \\
\hline if & 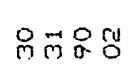 & 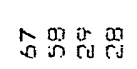 & 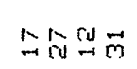 & orNNM & $\underset{\sim}{\infty} \hat{N}_{\sim}^{\infty}$ & 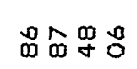 & 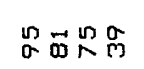 & 걱 & 슴욤유 & 果告类 \\
\hline 缺岁 & 品然家 & 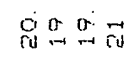 & $\dot{0} 0.0$ & rii ri ri ru & 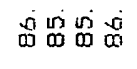 & 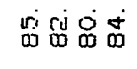 & 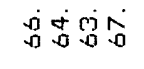 & 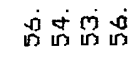 & 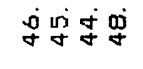 & m命官占 \\
\hline & 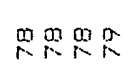 & 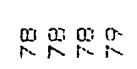 & 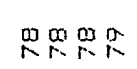 & 只果只 & 里里界只 & 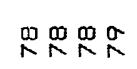 & 象黑只尗 & 象界员员 & 只品品只 & 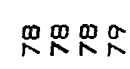 \\
\hline & $\Rightarrow$ curduru & 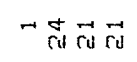 & 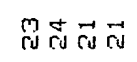 & 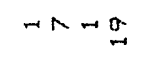 & 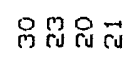 & 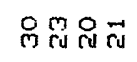 & 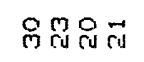 & 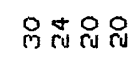 & 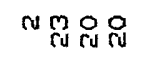 & " ్ָָก \\
\hline 岁 & 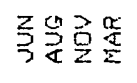 & 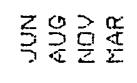 & 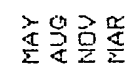 & 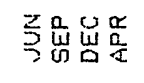 & 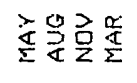 & 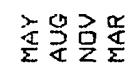 & 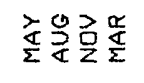 & $\frac{2}{2} \frac{3}{2} \frac{3}{2} \frac{\alpha}{2}$ & 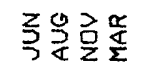 & 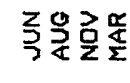 \\
\hline 㟒 & $\infty$ & $a$ & $m$ & $\stackrel{\mathscr{q}}{\forall}$ & 움 & 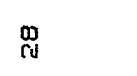 & $\underset{-1}{+}$ & + & $\overrightarrow{i n}$ & $\vec{q}$ \\
\hline 岁点 & vi & ñ & $\stackrel{n}{n}$ & $\stackrel{\mathfrak{m}}{\mathfrak{M}}$ & 罸 & 嵒 & 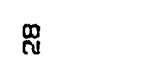 & "ִ & $\hat{\imath}$ & â \\
\hline 总总 & $\stackrel{m}{\wedge}$ & 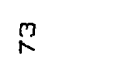 & $\stackrel{m}{n}$ & $\stackrel{m}{n}$ & $\stackrel{m}{n}$ & $\stackrel{M}{R}$ & $\stackrel{m}{n}$ & $\stackrel{m}{N}$ & $\stackrel{m}{R}$ & $\stackrel{m}{R}$ \\
\hline 㟧 & $\hat{n}$ & m & 点 & $\infty$ & 罚 & $\stackrel{q}{q}$ & $\stackrel{\infty}{\rightarrow}$ & $\stackrel{\mathbb{D}}{\mathfrak{m}}$ & - & กู \\
\hline 结兘 & $\vec{\sigma}$ & 웅 & 웅 & $\stackrel{g}{m}$ & $\stackrel{0}{+}$ & $\stackrel{n}{+}$ & J & g & $\stackrel{M}{q}$ & q \\
\hline 承㟧 & 움 & q & 웅 & $\stackrel{9}{q}$ & 웅 & 웅 & \& & 웅 & 웅 & 웅 \\
\hline$\frac{\dot{\rho}}{2}$ & $\begin{array}{l}\text { Oे } \\
\text { rud } \\
\text { ru }\end{array}$ & $\underset{r u}{r d u}$ & $\begin{array}{l}r u \\
r u \\
r d \\
r\end{array}$ & 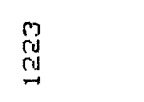 & $\underset{\vec{m}}{\vec{m}}$ & 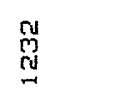 & 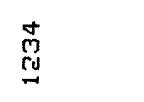 & $\stackrel{\stackrel{m}{9}}{\stackrel{M}{N}}$ & 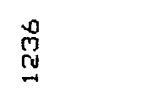 & 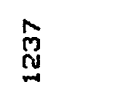 \\
\hline$\frac{\vec{u}}{3}$ & $z$ & $z$ & $z$ & $z$ & $z$ & $z$ & $z$ & $z$ & $z$ & $z$ \\
\hline
\end{tabular}




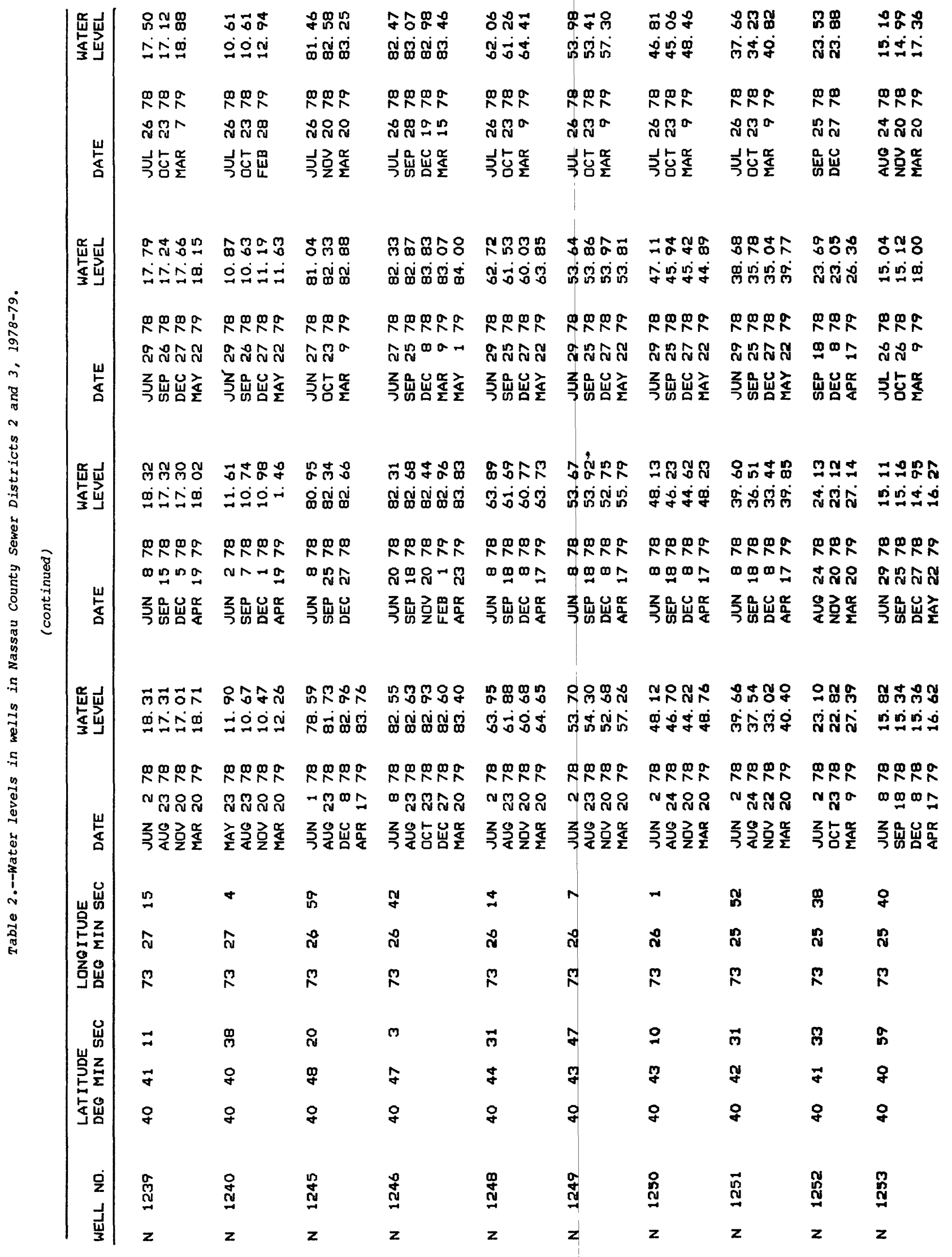




\begin{tabular}{|c|c|c|c|c|c|c|c|c|c|}
\hline 営岕 & 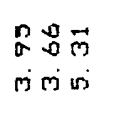 & 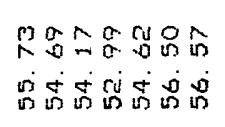 & 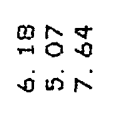 & 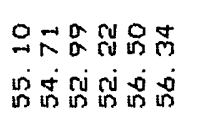 & 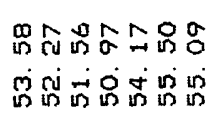 & $\begin{array}{l}\text { Mo } \\
\text { on } \\
0\end{array}$ & $\begin{array}{l}\text { 品 } \overrightarrow{0} \\
\text { mं子 }\end{array}$ & $\begin{array}{l}\text { लेठ丿 } \\
\text { लंभं लं }\end{array}$ & $\begin{array}{l}\text { gon } \\
\text { कino }\end{array}$ \\
\hline & $\stackrel{p}{\wedge} \stackrel{0}{\wedge}$ & 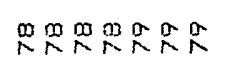 & $\stackrel{m}{\wedge} \frac{m}{n}$ & 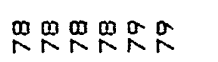 & 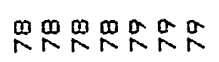 & $\stackrel{\mathscr{Q}}{\wedge} \stackrel{\mathscr{R}}{\wedge}$ & 罟界 & 果是足 & $\stackrel{\infty}{\wedge}$ \\
\hline & 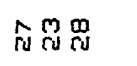 & 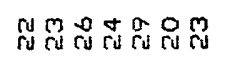 & तu rú & तु तु हैं & 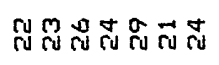 & rư & กับ & त्रत्रत्र & กั \\
\hline 崖 & 方志总 & 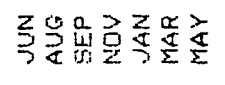 & 守皆造 & 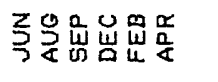 & 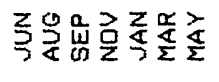 & 象峜 & 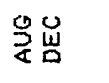 & $\frac{9}{4} \frac{0}{2} \frac{\alpha}{2}$ & 㩆 \\
\hline 䍃岕 & 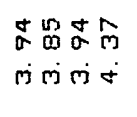 & 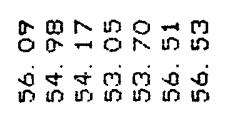 & 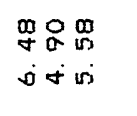 & 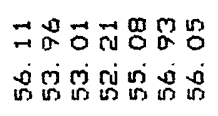 & 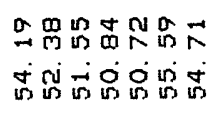 & $\begin{array}{l}\vec{\sigma} \vec{二} \vec{m} \\
\dot{0} \dot{0}\end{array}$ & 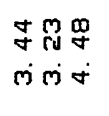 & 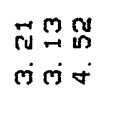 & 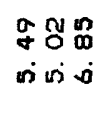 \\
\hline & 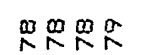 & 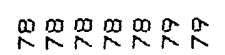 & 象界界 & 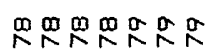 & 只只只只界卡の & m & $\stackrel{m}{\wedge} \stackrel{0}{r}$ & $\stackrel{m}{\wedge} \stackrel{a}{\wedge}$ & 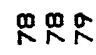 \\
\hline & 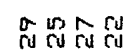 & 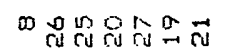 & 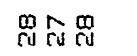 & 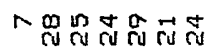 & 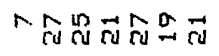 & กิญี กั & กิतี กั & กิ กี่ & ลิกีั \\
\hline 索 & 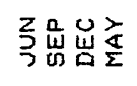 & 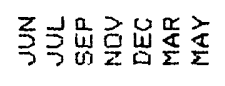 & 忿品出 & 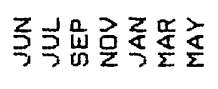 & 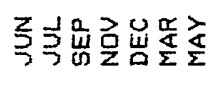 & 引豆充 & 古豆妾 & 引ち皆 & 引学京 \\
\hline 䍃耑 & 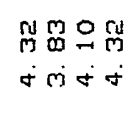 & 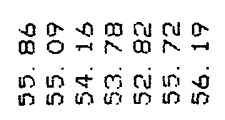 & 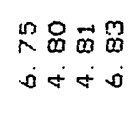 & 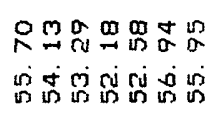 & 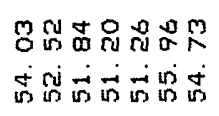 & 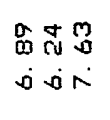 & 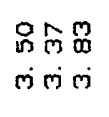 & 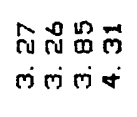 & 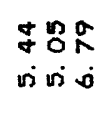 \\
\hline & 哭罣唄是 & 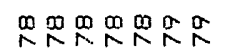 & 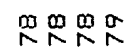 & 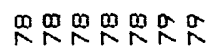 & 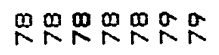 & $\stackrel{p}{\infty} \frac{0}{R}$ & 果是卡 & 勨界卡 & $\stackrel{\infty}{\wedge} \frac{a}{R}$ \\
\hline & ーNー㮩 & 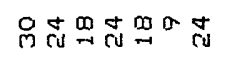 & $-\infty+\sigma$ & 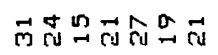 & 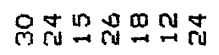 & 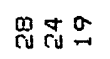 & तैं山्व & 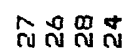 & พื้กำ \\
\hline 崖 & 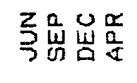 & 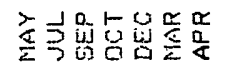 & 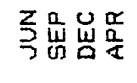 & 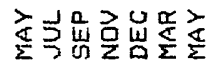 & 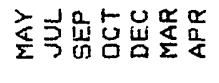 & 冚皆品 & 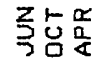 & 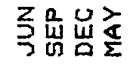 & 坫占竞 \\
\hline 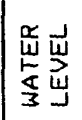 & 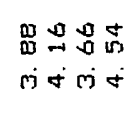 & 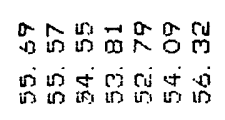 & 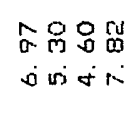 & 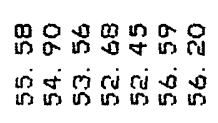 & 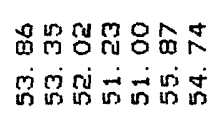 & 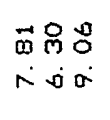 & 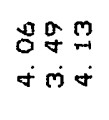 & 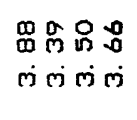 & 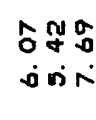 \\
\hline & $\stackrel{\text { pa }}{\wedge}$ & 累思是是是 & 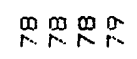 & 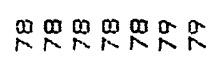 & 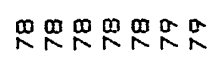 & $\stackrel{p}{R} \stackrel{a}{R}$ & 只果の & 象品果只 & mon \\
\hline & 品踏品 & a rang & 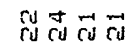 & 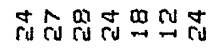 & 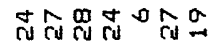 & 可品品 & 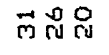 & $\vec{m}^{n-a}$ & - జี ชู \\
\hline 崖 & 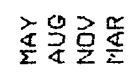 & 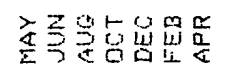 & 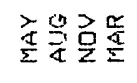 & 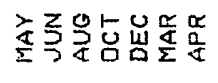 & 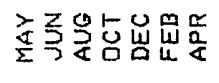 & 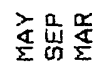 & 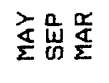 & 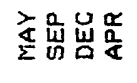 & 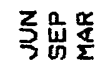 \\
\hline 岁 & กี & \pm & $\sigma$ & $\stackrel{9}{-1}$ & $\stackrel{\infty}{\infty}$ & $\vec{m}$ & $\stackrel{n}{n}$ & $\stackrel{n}{\sim}$ & m \\
\hline$\underset{\substack{T \\
0}}{2}$ & 品 & คิ & $\stackrel{\circ}{\leftarrow}$ & ते & ते & ले & $\stackrel{M}{M}$ & $\stackrel{M}{m}$ & 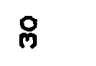 \\
\hline 造出 & $R^{2}$ & $m$ & $\stackrel{m}{n}$ & $\stackrel{m}{n}$ & $\stackrel{m}{\wedge}$ & 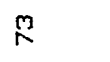 & 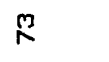 & 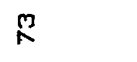 & $R$ \\
\hline 嵌 & $\because$ & a & $\hat{q}$ & $\mathscr{0}$ & c & के & 点 & in & - \\
\hline$\sum^{2}$ & q & $\stackrel{\text { 감 }}{2}$ & $\stackrel{m}{m}$ & gy & $\stackrel{\mathfrak{y}}{\forall}$ & $\stackrel{a}{m}$ & $\underset{m}{\mathfrak{m}}$ & $\stackrel{m}{m}$ & \& \\
\hline 疋出 & $g$ & 앙 & 웅 & 욤 & 웅 & 웜 & 웅 & 웅 & 웅 \\
\hline & 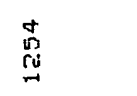 & 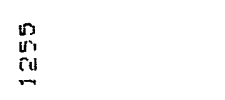 & $\hat{n}$ & 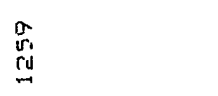 & $\begin{array}{l}\text { g } \\
\text { cu } \\
\text { cu }\end{array}$ & $\begin{array}{l}\sigma \\
\text { o } \\
\text { rid } \\
\text { r }\end{array}$ & $\overrightarrow{\widehat{N}}$ & 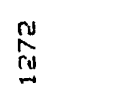 & 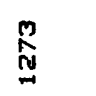 \\
\hline & $z$ & $z$ & $z$ & $z$ & $z$ & $z$ & $z$ & $z$ & $z$ \\
\hline
\end{tabular}




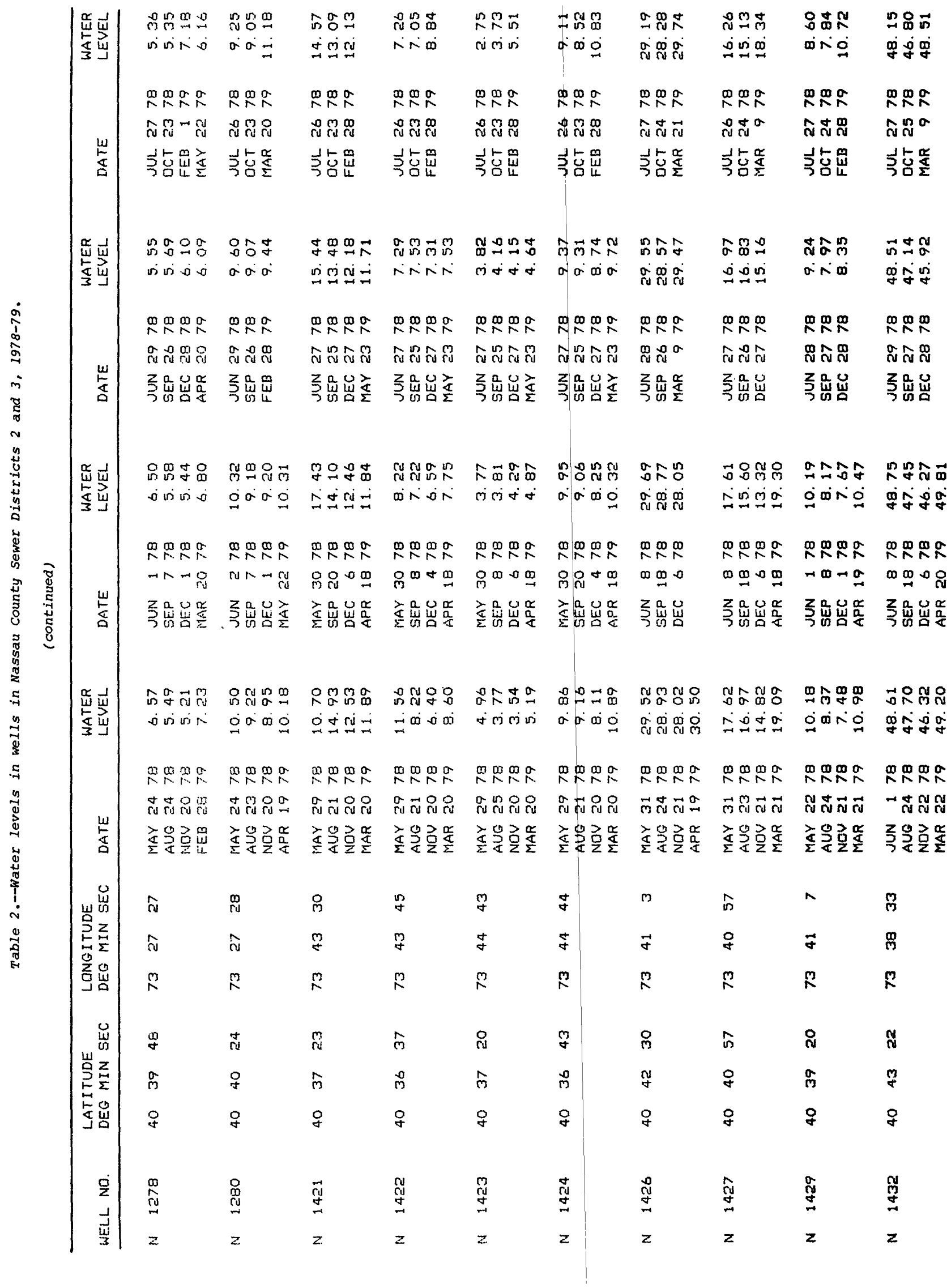




\begin{tabular}{|c|c|c|c|c|c|c|c|c|c|c|c|}
\hline 妥岃 & 吕鬲哭 & 品品 & $\stackrel{\substack{\infty \\
\infty}}{\stackrel{m}{\sim}}$ & 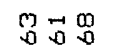 & 㟔 & 墭 & 总嵒品 & Mㅇㅇㅇ & 余嵒骂 & 息合紊 & 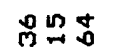 \\
\hline 兵出 & 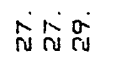 & mid & 舫 & 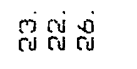 & $\neq 0$ & $\stackrel{\dot{m}}{-\dot{-}}$ & 0 ०० & மंग & ن̛ & $=\dot{a c j}$ & $\dot{+} \dot{+}$ \\
\hline & $\stackrel{m}{\wedge}$ & $\stackrel{n}{N} \stackrel{0}{N}$ & 思足 & $\stackrel{0}{R} R$ & $\stackrel{m}{R} \stackrel{p}{\wedge}$ & $\stackrel{\infty}{\wedge}$ & 畧只R & 只R & $\stackrel{p}{R} R^{0}$ & mag & $\stackrel{\infty}{\wedge} \frac{0}{R}$ \\
\hline & rog & ru & cid & 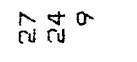 & ֻ̆ & cid & กิ่ & 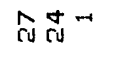 & 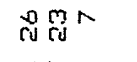 & ชุำ & 莎山 \\
\hline 崖 & $35 \frac{x}{5}$ & 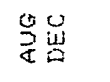 & 我品 & $35 \frac{\alpha}{3}$ & 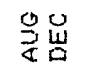 & 邹总 & 引占告 & $5 ち \frac{\alpha}{5}$ & 引ち采 & 引ち选 & 引号哭品 \\
\hline 受㟧 & $\log _{0 \rightarrow 0}^{0}$ & $\underset{\sim}{\infty}$ & $6 ?$ & 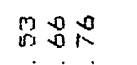 & 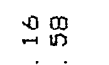 & 용. & M罗品 & 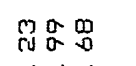 & 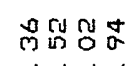 & 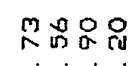 & ติตั \\
\hline 起出 & किति ति & 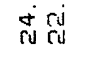 & $\rightarrow \infty$ & đår cuj cuj & $\stackrel{\infty}{\rightarrow \infty}$ & $\stackrel{m}{\rightarrow-1}$ & Nos & 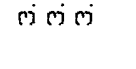 & 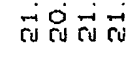 & 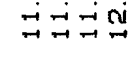 & $\dot{\sigma}$ हं \\
\hline & $\stackrel{m}{\wedge} \stackrel{p}{\wedge}$ & $\stackrel{p}{\wedge} \stackrel{0}{\wedge}$ & $\stackrel{m}{\wedge}$ & $\stackrel{p}{\wedge} \stackrel{p}{\wedge} \stackrel{0}{R}$ & $\stackrel{p}{\wedge} \underset{\wedge}{n}$ & m & $\stackrel{p}{n} \stackrel{p}{\wedge} \underset{\wedge}{n}$ & 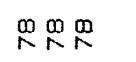 & m舟员朵 & mon & man \\
\hline & 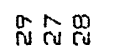 & ru & Nu & 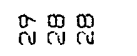 & ते ते & $\hat{\imath} \overrightarrow{r u}$ & 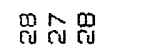 & ขึล & 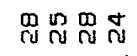 & 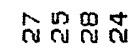 & กิํํำ \\
\hline 崖 & 强品 & 马를 & 3 & 强品 & 方总 & 占学 & 总㟧㟒 & 引品㟧 & 学出出察 & 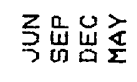 & 引皆题 \\
\hline 吕 & 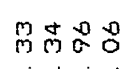 & ${ }_{\rightarrow \rightarrow}=\vec{B}$ & 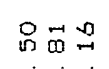 & 称品 & Mas & 品品二 & 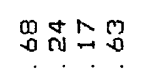 & ज合灾 & 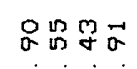 & 寸贺只二 & 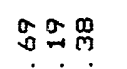 \\
\hline 安勇 & 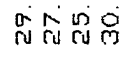 & ñ & $\stackrel{0}{\sim}$ & $\begin{array}{l}\text { nirj rid ro } \\
\text { cu ru ru }\end{array}$ & $\underset{n}{0}$ & $\stackrel{9}{\rightarrow}$ & ríó & लं & तं तี & $\stackrel{n j}{=}=\dot{\tau}$ & $\dot{\theta}+\dot{i n}$ \\
\hline & 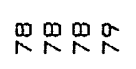 & $\stackrel{m}{\wedge} \stackrel{\infty}{\wedge}$ & $\stackrel{m}{n} \stackrel{\infty}{\wedge}$ & 只界只只 & $\stackrel{m}{\wedge} \stackrel{p}{R}$ & 品只只 & 象只员界 & 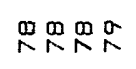 & 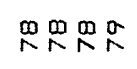 & 番采品 & ロ゚゚ \\
\hline & $\infty \infty_{-1}^{\infty} 0_{\text {ru }}$ & 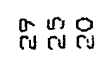 & $\underset{r i d}{a}$ 出 & $m \underset{r}{\infty} 0$ & 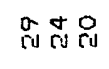 & 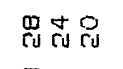 & $-\infty-g$ & $+\infty-g$ & $\cos 00$ & $\cos \theta$ & 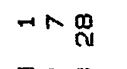 \\
\hline 峔 & 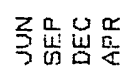 & $\begin{array}{l}350 \\
3 \\
3\end{array}$ & 3 & 引品岩哭 & 引。ㅣㅁ & 引ち骂 & 引号㟔品 & 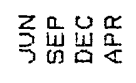 & 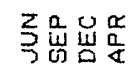 & 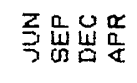 & 活品斀 \\
\hline 営 & ति & $\begin{array}{l}m \pm 0 \\
0\end{array}$ & 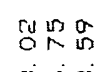 & 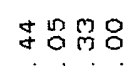 & 品合品 & ํㅗㅇㅁㅇㅁ & मूल & 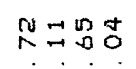 & 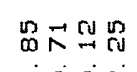 & 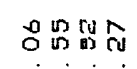 & 정웅움욤 \\
\hline 秀出 & 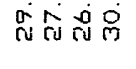 & rug ricú & 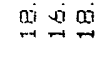 & 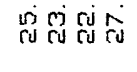 & ario & 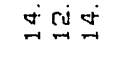 & $\wedge 0$ ம & मेंr rí & 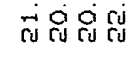 & 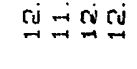 & मिं लिक \\
\hline & 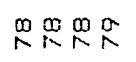 & 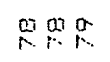 & 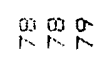 & 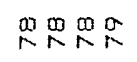 & pog & 界界帜 & 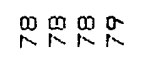 & 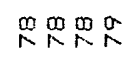 & 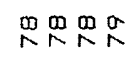 & $\stackrel{m}{\wedge} \operatorname{mog}_{\wedge}^{\infty}$ & 急象 \\
\hline & 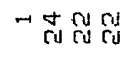 & 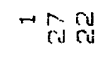 & $-\mathrm{rud}$ & - & $-7 x$ rd & $-\hat{r u c y}$ & 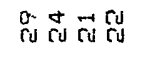 & rud ru ru rud & 표 & 음유 & ตํํำ \\
\hline 岁 & $\begin{array}{l}z \\
3\end{array}$ & 总䖵告 & 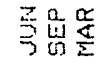 & $\begin{array}{l}2 \\
3 \\
3\end{array}$ & 引品告 & 枵㟧告 & 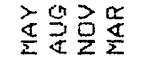 & 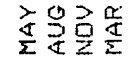 & 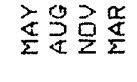 & 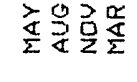 & 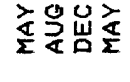 \\
\hline 岂 & $\stackrel{0}{m}$ & 落 & $\exists$ & $\stackrel{0}{-1}$ & + & 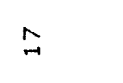 & 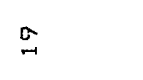 & $\stackrel{r u}{\sim}$ & 0 & $\stackrel{N}{\rightarrow}$ & $\stackrel{N}{\sim}$ \\
\hline$\frac{2}{2}$ & 搹 & 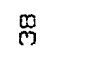 & 8 & $\stackrel{m}{m}$ & 吕 & 思 & 号 & 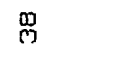 & m & $\stackrel{n}{m}$ & ตั \\
\hline 营总 & $m^{3}$ & 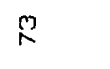 & 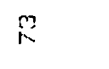 & $\stackrel{n}{R}$ & $\stackrel{M}{n}$ & $\stackrel{M}{R}$ & $\stackrel{m}{n}$ & $M$ & $\stackrel{M}{n}$ & m & $\stackrel{9}{R}$ \\
\hline 㟧 & d & $\infty$ & 9 & 0 & $a$ & ru & $\stackrel{0}{a}$ & in & חָ & $\sigma$ & $m$ \\
\hline 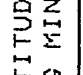 & $\vec{\sigma}$ & $\vec{q}$ & 9 & $\vec{t}$ & $q$ & $\stackrel{a}{\mathrm{c}}$ & $\stackrel{\infty}{m}$ & $\stackrel{m}{m}$ & 움 & $\stackrel{a}{n}$ & $\stackrel{\sigma}{m}$ \\
\hline 氞总 & $\stackrel{\circ}{\circ}$ & q & 9 & 움 & 워 & $q$ & \& & $\stackrel{\circ}{4}$ & 움 & $\stackrel{\circ}{q}$ & q \\
\hline$\frac{9}{2}$ & 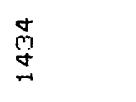 & $\begin{array}{l}\sqrt[n]{9} \\
\stackrel{9}{4}\end{array}$ & 萑 & $\begin{array}{l}\hat{n} \\
\substack{c \\
\square}\end{array}$ & 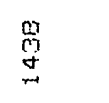 & 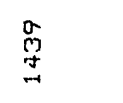 & 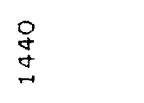 & $\underset{+}{\vec{d}}$ & 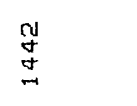 & 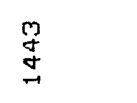 & $\underset{J}{d}$ \\
\hline$\frac{\vec{u}}{3}$ & $z$ & $z$ & 2 & $z$ & $z$ & $z$ & $z$ & $z$ & $z$ & $z$ & $z$ \\
\hline
\end{tabular}




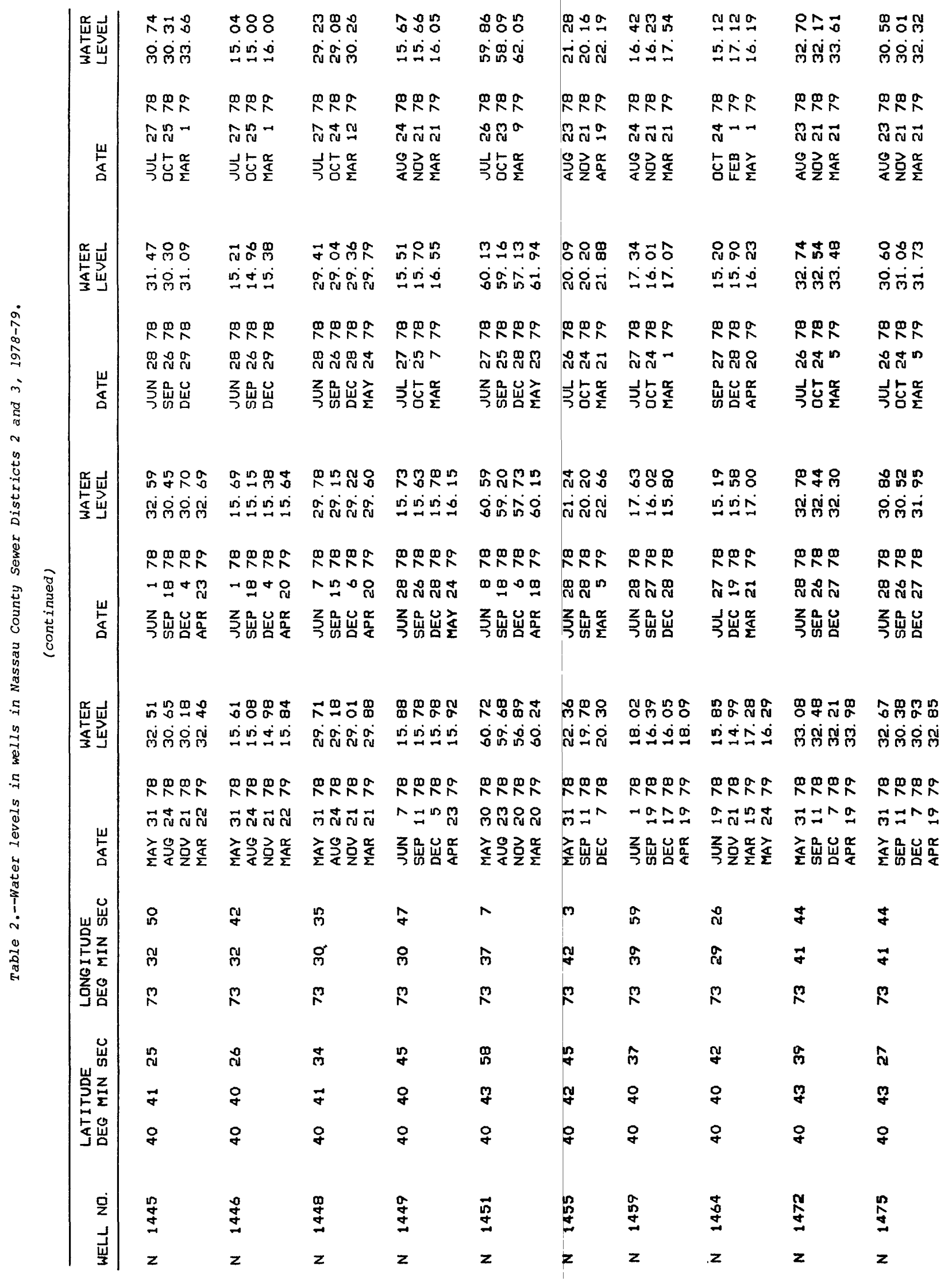




\begin{tabular}{|c|c|c|c|c|c|c|c|c|c|c|}
\hline 岳崖 & 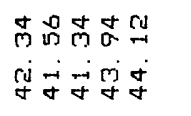 & 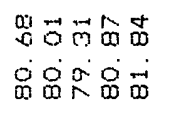 & 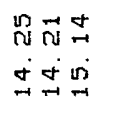 & 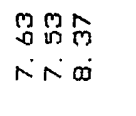 & $\begin{array}{l}n=0 \\
n \infty 00 \\
n+\infty\end{array}$ & 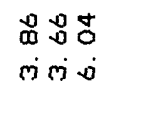 & $\begin{array}{l}\text { min } \\
\text {-io }\end{array}$ & $\begin{array}{l}80 \\
0 \% \\
00\end{array}$ & 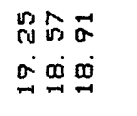 & 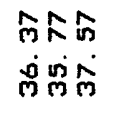 \\
\hline & 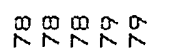 & 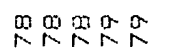 & $\stackrel{m}{\wedge} \stackrel{\infty}{\wedge}$ & $\stackrel{p}{\wedge} R^{\infty}$ & $\stackrel{p}{\wedge} \stackrel{p}{R}$ & $\stackrel{m}{\wedge}$ & $\stackrel{\mathbb{R}}{\wedge}$ & $\stackrel{R}{R}$ & $\stackrel{R}{\wedge} R^{\infty}$ & 果员员 \\
\hline & 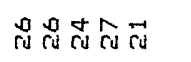 & 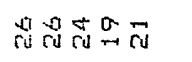 & תִ & 모윰요 & 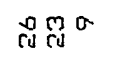 & 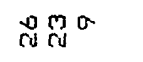 & חָ & ç กิ & 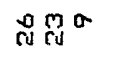 & กิतू \\
\hline 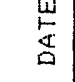 & 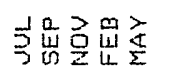 & 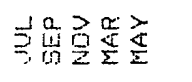 & $5 \frac{\alpha}{3}$ & 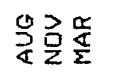 & 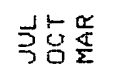 & $\frac{1}{5} \frac{6}{5}$ & 引㟧 & 引品 & $35 \frac{\alpha}{3}$ & 引占䍃 \\
\hline 品 & 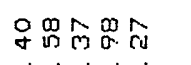 & 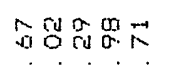 & 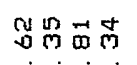 & 뭉뭉 & 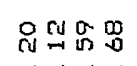 & 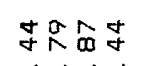 & 岗 & 艁嵒 & 品西哭 & 몽요 \\
\hline 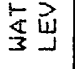 & 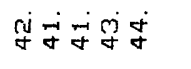 & $\operatorname{cis} \frac{0}{\pi}$ & $\stackrel{+}{\sim} \dot{\sim}$ & $\wedge \dot{0}$ & कintr & 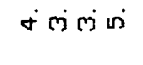 & -io & -iri & $\underset{\sigma}{\sigma} \underset{\sim}{\infty} \underset{\sim}{\infty} \sigma$ & mं m户 m户 \\
\hline & 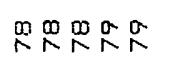 & 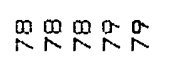 & 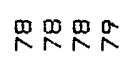 & 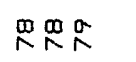 & 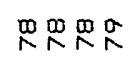 & 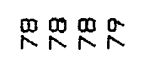 & $\stackrel{\mathbb{R}}{R} \mathbb{R}$ & $\stackrel{m}{\stackrel{m}{N}}$ & 品界畧。 & 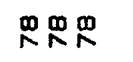 \\
\hline & 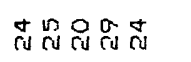 & 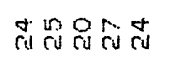 & 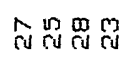 & 은 & 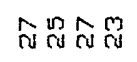 & 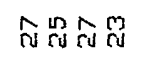 & $\hat{c u} \stackrel{n}{\text { of }}$ & $\hat{n}^{\circ}$ & 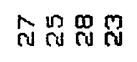 & ณึก กิก \\
\hline 㟶 & 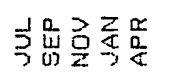 & 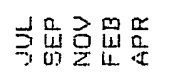 & 豆品㟔这 & $35 \frac{\alpha}{5}$ & 枵品㟧度 & 强㟧崖 & 至品 & 引号 & 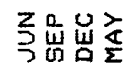 & 弓出㟧 \\
\hline 品 & 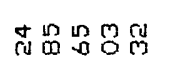 & 可苨品品员 & 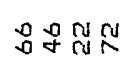 & 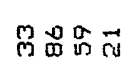 & 的致合品 & 致品 & Cy & is g & 孚眾嵒占 & 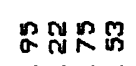 \\
\hline 岂 & 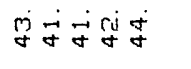 & $8 R$ & 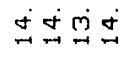 & $\operatorname{mrn}$ & $\dot{\theta} \boldsymbol{\theta} \dot{r}$ & ம் & -iriri & $-i x$ & $\underset{\sigma}{\sigma} \underset{\sim}{0} \underset{\sim}{\infty}$ & ค่ \\
\hline & 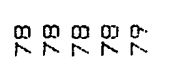 & 品界界 & 黑只界只 & 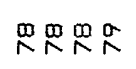 & m番品 & 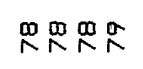 & maga & $\stackrel{\mathbb{m}}{\wedge} \stackrel{\mathbb{R}}{\wedge}$ & 只只界尔 & 果果果只 \\
\hline & त्य & तu & $\infty \underset{\sim}{\infty} \stackrel{\infty}{\sim}$ & 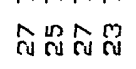 & $\infty \underset{\sim}{\infty} \sim \infty$ & $\infty \underset{\sim}{\infty} \sim \underset{\sim}{\infty}$ & g w & $p^{\infty}$ & $\boldsymbol{\infty} \underset{\sim}{\infty} \underset{\sim}{m}$ & $\infty \ddot{0} 0$ \\
\hline 崖 & 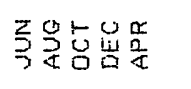 & 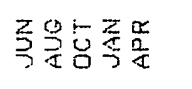 & 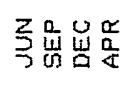 & 强㟧充 & 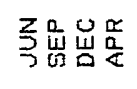 & 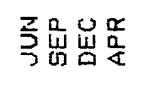 & 空品品 & 䆜品 & 豆品岩竞 & 枵品㟧喿 \\
\hline$\vec{v}$ & 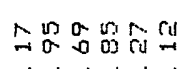 & 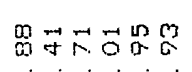 & 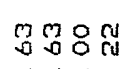 & 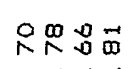 & 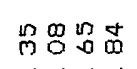 & 선 & 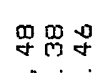 & $\stackrel{0}{=} \vec{\infty} \vec{m}$ & 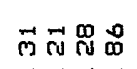 & M品ล视 \\
\hline & 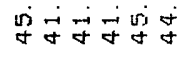 & gidnog & 导导导导 & 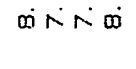 & $\dot{0} 0$ & में कं & $\Rightarrow \dot{\theta}$ & si & 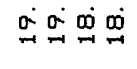 & ติ่ต் \\
\hline & 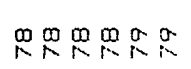 & 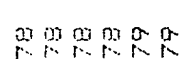 & 舟界照朵 & mag & a & 象塄品员 & $\stackrel{\infty}{\wedge} \underset{\wedge}{\wedge}$ & $\stackrel{p}{R}$ & m里员尔 & 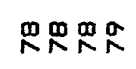 \\
\hline & 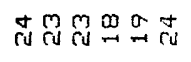 & 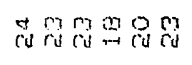 & 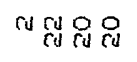 & 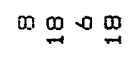 & 品屾品 & 品品品品 & ơd & 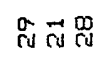 & 品药踣 & ตึณี กี \\
\hline 崖 & 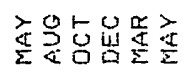 & 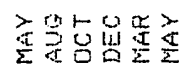 & $30 \frac{0}{3}$ & 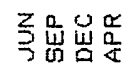 & 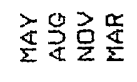 & 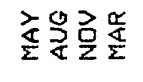 & 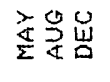 & 空定罢 & 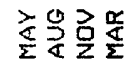 & 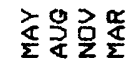 \\
\hline 岁 & 0 & $\stackrel{\sim}{\sim}$ & $g$ & $\stackrel{a}{a}$ & $\stackrel{+}{m}$ & 罚 & 요 & ⿵人 & 离 & I \\
\hline 5 & $\overleftarrow{m}$ & $m$ & 9 & $\stackrel{M}{4}$ & $\stackrel{m}{4}$ & $\stackrel{9}{4}$ & $\stackrel{m}{q}$ & $\stackrel{\mathfrak{Y}}{y}$ & $\stackrel{q}{\sigma}$ & 7 \\
\hline 总㟧 & $\stackrel{M}{\wedge}$ & 9 & n & $n$ & $\stackrel{m}{n}$ & $M$ & $R^{3}$ & $m$ & $\stackrel{M}{R}$ & $R$ \\
\hline 岁 & $a$ & I & $\stackrel{a}{-1}$ & $\stackrel{g}{m}$ & $\stackrel{\circ}{q}$ & a & $\infty$ & + & $\exists$ & กิ \\
\hline 胥焉 & $\stackrel{c u}{4}$ & $\stackrel{r a}{y}$ & gr & y & 움 & $\stackrel{a}{a}$ & 点 & $\stackrel{m}{m}$ & 9 & m \\
\hline 氙㟔 & $g$ & 8 & if & q & 움 & q & 웅 & 9 & 움 & q \\
\hline$\frac{1}{2}$ & $\stackrel{n}{\overrightarrow{0}}$ & $\frac{0}{0}$ & 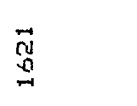 & $\begin{array}{l}\text { ru } \\
\text { Nu } \\
0 \\
-1\end{array}$ & 点 & $\begin{array}{l}\stackrel{0}{N} \\
\substack{0 \\
=}\end{array}$ & 垈 & $\begin{array}{l}0 \\
\text { cij } \\
0 \\
0 \\
-1\end{array}$ & $\begin{array}{l}c u \\
0 \\
0 \\
0 \\
0-1\end{array}$ & 㩊 \\
\hline 荘 & $z$ & $z$ & $z$ & $z$ & $z$ & $z$ & $z$ & $z$ & $z$ & $z$ \\
\hline
\end{tabular}




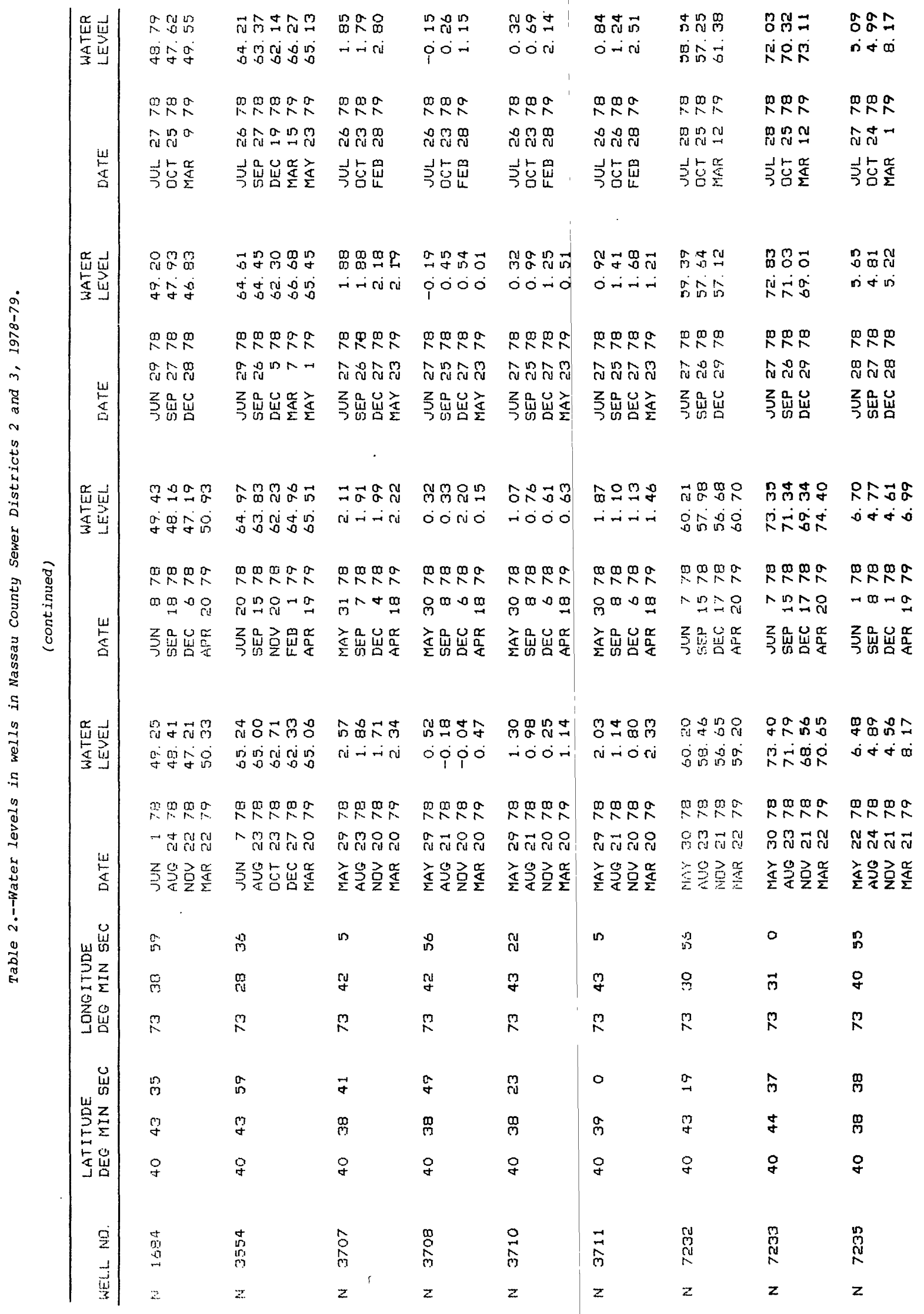




\begin{tabular}{|c|c|c|c|c|c|c|c|c|c|c|}
\hline 嵒岀 & $\begin{array}{l}\text { Uu } \\
50 \\
50\end{array}$ & 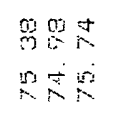 & 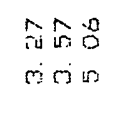 & 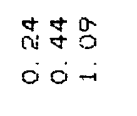 & $\begin{array}{l}\text { mo } \\
0.0 \\
\wedge i \infty\end{array}$ & 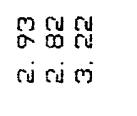 & 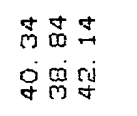 & 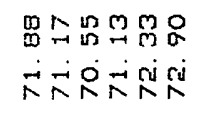 & 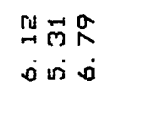 & 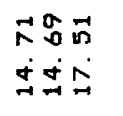 \\
\hline & $a$ & 里只 & 果只 & apd & $\stackrel{\infty}{R}$ & gar & 象RR & man & $\stackrel{0}{\wedge} \wedge$ & 象昗尔 \\
\hline & $\ddot{\mathrm{ru}}$ & $90^{\circ}$ & is 20 & तu & rid & pog & 总 & 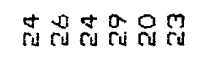 & du & ลูก \\
\hline 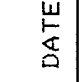 & $\frac{x}{3}$ & 3 & 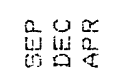 & 少它罟 & 㽞 & 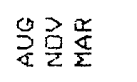 & 歺皆童 & 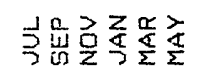 & $35 \frac{\alpha}{5}$ & 引ち匕 \\
\hline 戦岂 & 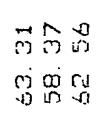 & 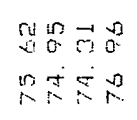 & $\begin{array}{l}\text { gino } \\
\text { ricin } \\
\text { ris }\end{array}$ & 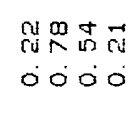 & $\begin{array}{l}\text { n:o } \\
0.0 \\
\infty 10\end{array}$ & 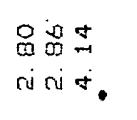 & 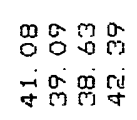 & 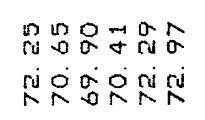 & 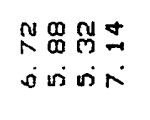 & 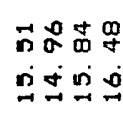 \\
\hline & 品果品 & mag & 足只R & 采品品员 & 员足 & $\stackrel{m}{n} \stackrel{0}{\wedge}$ & $\stackrel{m a g}{x}$ & mpmón & 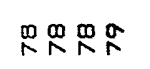 & 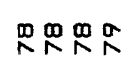 \\
\hline & $\mathrm{nat}$ & arru & rubat & 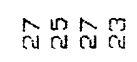 & तิ તี & वृp & 嵒识品罚 & 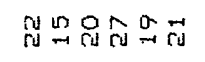 & กินกำ & 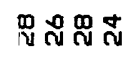 \\
\hline $\begin{array}{l}\text { 岁 } \\
a \\
\text { 口 }\end{array}$ & 至品立 & 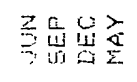 & 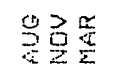 & 引号品充 & $\frac{1}{3}$ & 起号思 & 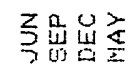 & 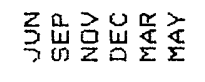 & 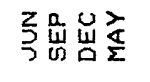 & 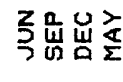 \\
\hline 䍃岂 & 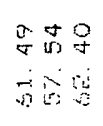 & 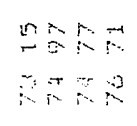 & $\begin{array}{l}900 \\
805 \\
605\end{array}$ & $\begin{array}{l}\mathscr{Q}-1-1 \\
\& \mathbb{N} \\
0000\end{array}$ & 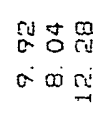 & 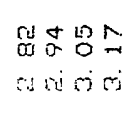 & 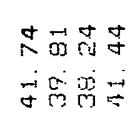 & 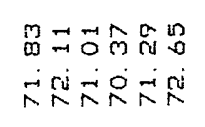 & 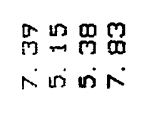 & 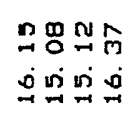 \\
\hline & 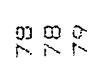 & man & 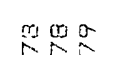 & 界品罢是 & $\stackrel{m}{R} \stackrel{\infty}{\wedge}$ & mp & 象界足 & mana & $\stackrel{m}{\wedge} \stackrel{\infty}{\wedge} \mathfrak{R}$ & 番界只 \\
\hline & $m \sqrt{3}$ & 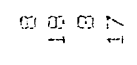 & $\mathrm{Min}$ & $g^{\infty} \backsim \pi$ & ru riो & 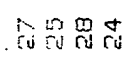 & $\operatorname{man} \rightarrow$ & 吅品哭人品 & $\underset{-1}{\infty} \sim \underset{\sim}{\infty}$ & Nmo \\
\hline 崖 & 驾望 & 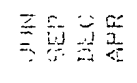 & $\frac{1}{3} \frac{2}{a}$ & 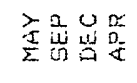 & 引紫竞 & 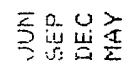 & 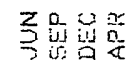 & 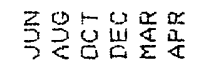 & 强品㟧器 & 枵品㟧品 \\
\hline 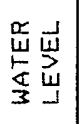 & 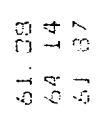 & 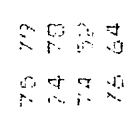 & 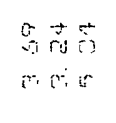 & 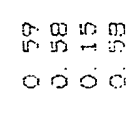 & 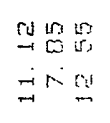 & 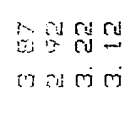 & 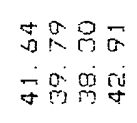 & 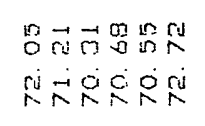 & 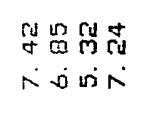 & 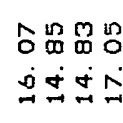 \\
\hline & $p 00$ & 9090 & 90 & 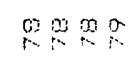 & 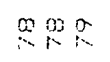 & 只品 & RR & mpgor & 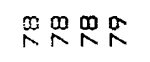 & $\stackrel{m}{\wedge} \wedge^{\infty} \stackrel{R}{\wedge}$ \\
\hline & 89 & अवि & $99^{\circ}$ & mand & $\begin{array}{cc}-1 \\
\text { ras }\end{array}$ & $\therefore \rightarrow \sigma$ & -9ि? & 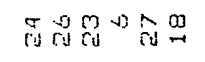 & 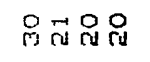 & 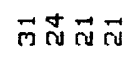 \\
\hline 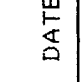 & 突采 & 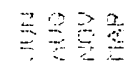 & 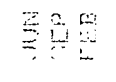 & 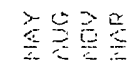 & 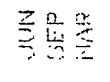 & 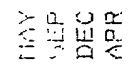 & 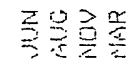 & 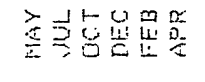 & 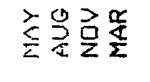 & 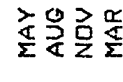 \\
\hline 岀 & $s$ & 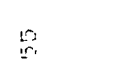 & 9 & 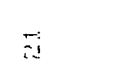 & $B$ & $\because$ & $\stackrel{?}{+}$ & 0 & $\theta$ & กั \\
\hline$\frac{z}{2}$ & 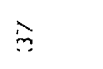 & $\because$ & $\ddot{E}$ & $\frac{9}{4}$ & $\&$ & 5 & 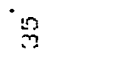 & 仓े & $\stackrel{M}{\leftarrow}$ & 욤 \\
\hline 总总 & $\approx$ & $n$ & 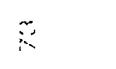 & $i^{2}$ & $R$ & $a$ & $m$ & $n$ & $R^{2}$ & $m^{m}$ \\
\hline 嵌 & $\stackrel{\infty}{-}$ & 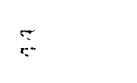 & 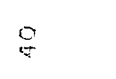 & $\stackrel{a}{\gtrless}$ & $\mathrm{m}$ & $a$ & $\mathrm{du}$ & $\stackrel{8}{2}$ & $\stackrel{\infty}{\sim}$ & $\stackrel{a}{m}$ \\
\hline 를 & $\dot{z}$ & 8 & $g$ & $\stackrel{g}{g}$ & $\stackrel{9}{q}$ & 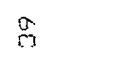 & $\stackrel{c y}{+}$ & $\stackrel{n}{+}$ & $\vec{F}$ & q \\
\hline 占㟔 & $\frac{9}{9}$ & 9 & $\ddot{\sigma}$ & 움 & 움 & g & \& & $g$ & 움 & q \\
\hline 是 & 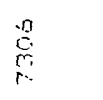 & 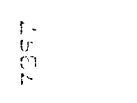 & $\begin{array}{c}0 \\
\substack{0 \\
\vdots \\
\vdots}\end{array}$ & 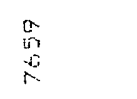 & 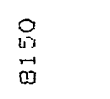 & 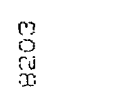 & $\begin{array}{l}n \\
c \\
c \\
0 \\
0\end{array}$ & $\begin{array}{l}0 \\
w \\
0 \\
0 \\
0\end{array}$ & 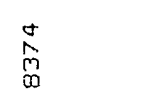 & 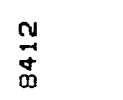 \\
\hline$\underline{4}$ & $z$ & $=$ & $z$ & $z$ & $\vec{z}$ & $\because$ & z & $z$ & $z$ & $z$ \\
\hline
\end{tabular}




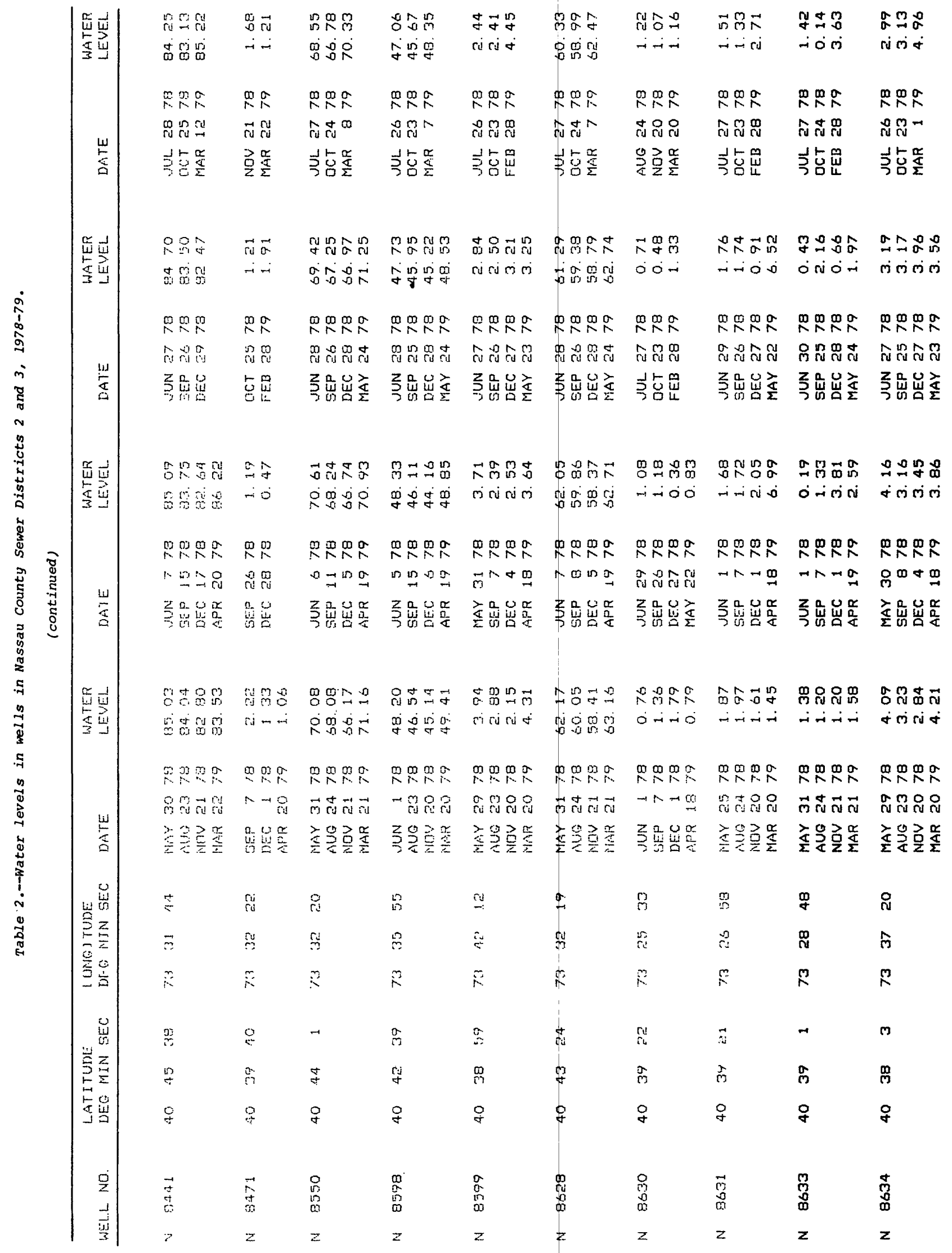




\begin{tabular}{|c|c|c|c|c|c|c|c|c|c|c|c|}
\hline 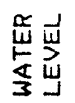 & 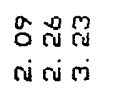 & $\begin{array}{l}30 \\
08 \\
00\end{array}$ & 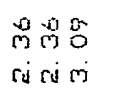 & 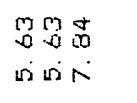 & 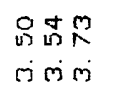 & $\begin{array}{l}0 \overrightarrow{0} \vec{m} \\
0-1 m \\
0-10\end{array}$ & $\begin{array}{l}0.05 \\
0.00 \\
-7-1\end{array}$ & 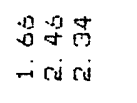 & 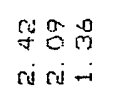 & 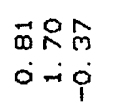 & 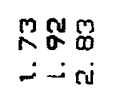 \\
\hline & $\stackrel{\infty}{\wedge} \stackrel{\infty}{\wedge}$ & $\stackrel{n}{\wedge}$ & man & 电只 & 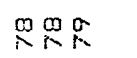 & $\cos$ & moR & $\stackrel{a R R}{R}$ & $\stackrel{p o g}{R}$ & man & $\stackrel{p}{\wedge} \stackrel{0}{n}$ \\
\hline & న̊ำ ભ & 番 & กิ & ㅇำ & 옹ㅁำ & ते & 華范品 & คูณ & $\wedge \overrightarrow{r u}$ तu & 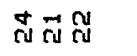 & กัญ ณี \\
\hline 崖 & 方皆采 & 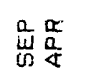 & 引占犎 & 古号品 & 乌占罟 & 南占告 & 인룰 & 引品 & 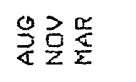 & $\frac{0}{4} \frac{0}{2} \frac{\pi}{2}$ & วち点 \\
\hline 离 & 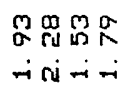 & 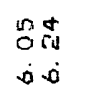 & 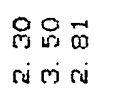 & 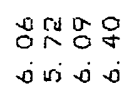 & 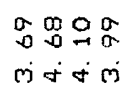 & 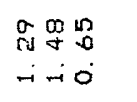 & $\begin{array}{l}\exists \sigma \alpha \\
7 \sigma \\
-i 0-\end{array}$ & 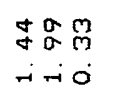 & 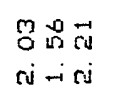 & 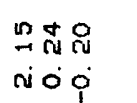 & 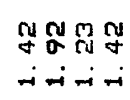 \\
\hline & 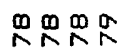 & 只只 & $\stackrel{m}{R}: \frac{\infty}{R}$ & 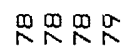 & 里员象の & $\stackrel{p}{\wedge} \mathbb{P}^{\infty}$ & $\mathbb{R}^{\infty} \frac{\infty}{R}$ & $\stackrel{\mathbb{R}}{\wedge} \stackrel{\mathbb{R}}{\wedge}$ & $\stackrel{m}{\wedge} \frac{a}{R}$ & $\stackrel{p}{\wedge} \stackrel{\infty}{\wedge}$ & 象是界 \\
\hline & ลิณ กิ ญิ & तु) & pu cu cu & 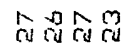 & กิ & 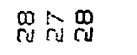 & $\hat{\text { Nơ }}$ & ำกำ & กิ号罟 & ล只思 & กิกลก \\
\hline 客 & 强㟧衣 & 号㟒 & 方品崫 & 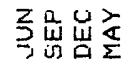 & 引品㟔充 & 引号㞻 & 古点品 & 强虽出 & 引点品 & 方占乳 & 枵恕出充 \\
\hline 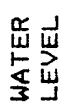 & 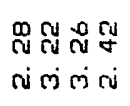 & $\begin{array}{l}\hat{N} \\
\text { in is }\end{array}$ & 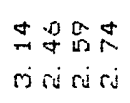 & 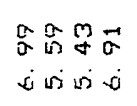 & 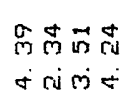 & 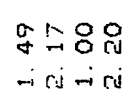 & 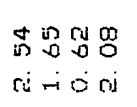 & 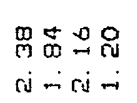 & $\begin{array}{l}=98 \\
=08 \\
-1-0\end{array}$ & 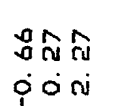 & 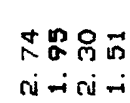 \\
\hline & mon & 政 & 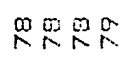 & mag & m里员品 & mpgra & mag & m番品员 & $\stackrel{p}{R} \stackrel{p}{R}$ & $\stackrel{\infty}{\wedge} \stackrel{\infty}{\wedge}$ & 象员员 \\
\hline & $\mathrm{p}^{\infty}+\stackrel{\Phi}{-}$ & 옷요 & $+\omega-0$ & 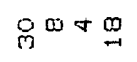 & $\stackrel{p}{m}^{\infty}+\underset{\sim}{\infty}$ & $\rightarrow \underset{-1}{a}-\sigma$ & 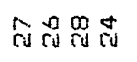 & $\vec{m}^{n-O}$ & 号点品 & 品塄䀡 & $\vec{m}^{N}+\sigma$ \\
\hline 占 & 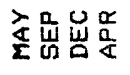 & 引つ & 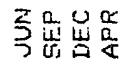 & 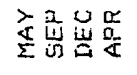 & 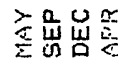 & 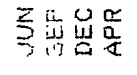 & 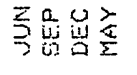 & 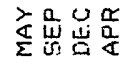 & 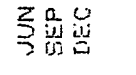 & 怒虽㟧 & 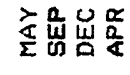 \\
\hline 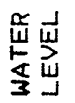 & 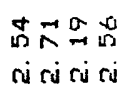 & 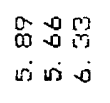 & 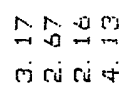 & 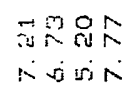 & 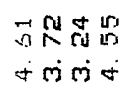 & 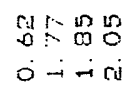 & 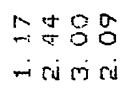 & 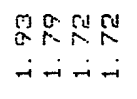 & 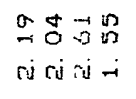 & 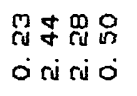 & 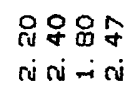 \\
\hline & 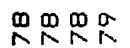 & $\underset{i}{\infty} \operatorname{mog}_{i}^{\infty}$ & $\underset{N}{m p d a g}$ & 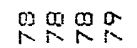 & man & 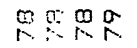 & ars & 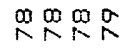 & 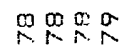 & 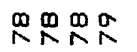 & m象品 \\
\hline & 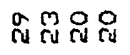 & A M M & 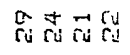 & 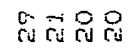 & 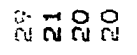 & 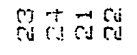 & $\vec{m}+\vec{q}$ & 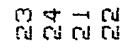 & 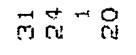 & $\vec{m}^{\wedge-0}$ ri & 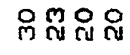 \\
\hline 桌 & 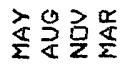 & 紊吉毫 & 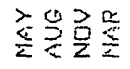 & 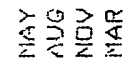 & 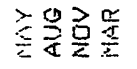 & 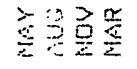 & 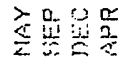 & 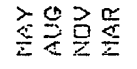 & 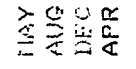 & 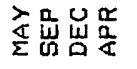 & 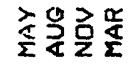 \\
\hline 㟧 & $\underset{\sim}{\infty}$ & id & $\underset{-1}{ \pm}$ & 0 & 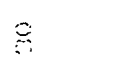 & 90 & $\underset{\alpha}{\alpha}$ & $\exists$ & 요 & 梁 & $\stackrel{9}{-1}$ \\
\hline $\bar{\Sigma}$ & $\hat{M}$ & $\stackrel{t}{\leftarrow}$ & $\stackrel{\infty}{\cdots}$ & 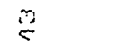 & 2 & $\tilde{F}$ & 萢 & $\vec{g}$ & $\stackrel{N}{*}$ & N & $\stackrel{0}{m}$ \\
\hline 㟧 & $\stackrel{M}{n}$ & $n^{2}$ & $m^{2}$ & $\ddot{R}$ & $\stackrel{2}{*}$ & $N$ & m & $n^{n}$ & $\mathrm{~m}^{3}$ & 2 & 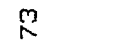 \\
\hline 荡 & ָे & $\overrightarrow{c u}$ & $\stackrel{+}{i s}$ & $\stackrel{M}{m}$ & 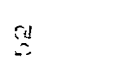 & $a$ & 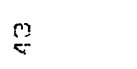 & 8 & $\stackrel{0}{-}$ & $\stackrel{n}{m}$ & in \\
\hline$\frac{3}{5}$ & $\hat{m}$ & $n$ & $\hat{m}$ & $m$ & $\stackrel{m}{n}$ & $\hat{m}$ & $\stackrel{m}{m}$ & $\stackrel{m}{g}$ & $\stackrel{0}{5}$ & m & $\hat{m}$ \\
\hline 我落 & $q$ & 웅 & 웅 & 舟 & 움 & $\stackrel{q}{q}$ & g & iq & 웅 & q & 우 \\
\hline$\dot{z}$ & $\begin{array}{l}\text { m } \\
\text { D } \\
\text { m }\end{array}$ & 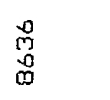 & $\begin{array}{l}\hat{\gamma} \\
0 \\
\infty\end{array}$ & $\begin{array}{l}\text { J } \\
\dot{0} \\
0 \\
\infty\end{array}$ & $\begin{array}{l}\stackrel{0}{0} \\
\stackrel{\oplus}{0} \\
\stackrel{0}{0}\end{array}$ & $\begin{array}{l}\hat{A} \\
\hat{1} \\
0 \\
0\end{array}$ & 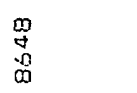 & 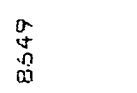 & $\begin{array}{l}\vec{n} \\
0 \\
0\end{array}$ & 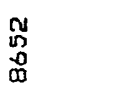 & 兑 \\
\hline$\frac{4}{3}$ & $z$ & $z$ & $z$ & $z$ & $=$ & $=r$ & $z$ & $z$ & $z$ & $z$ & $z$ \\
\hline
\end{tabular}




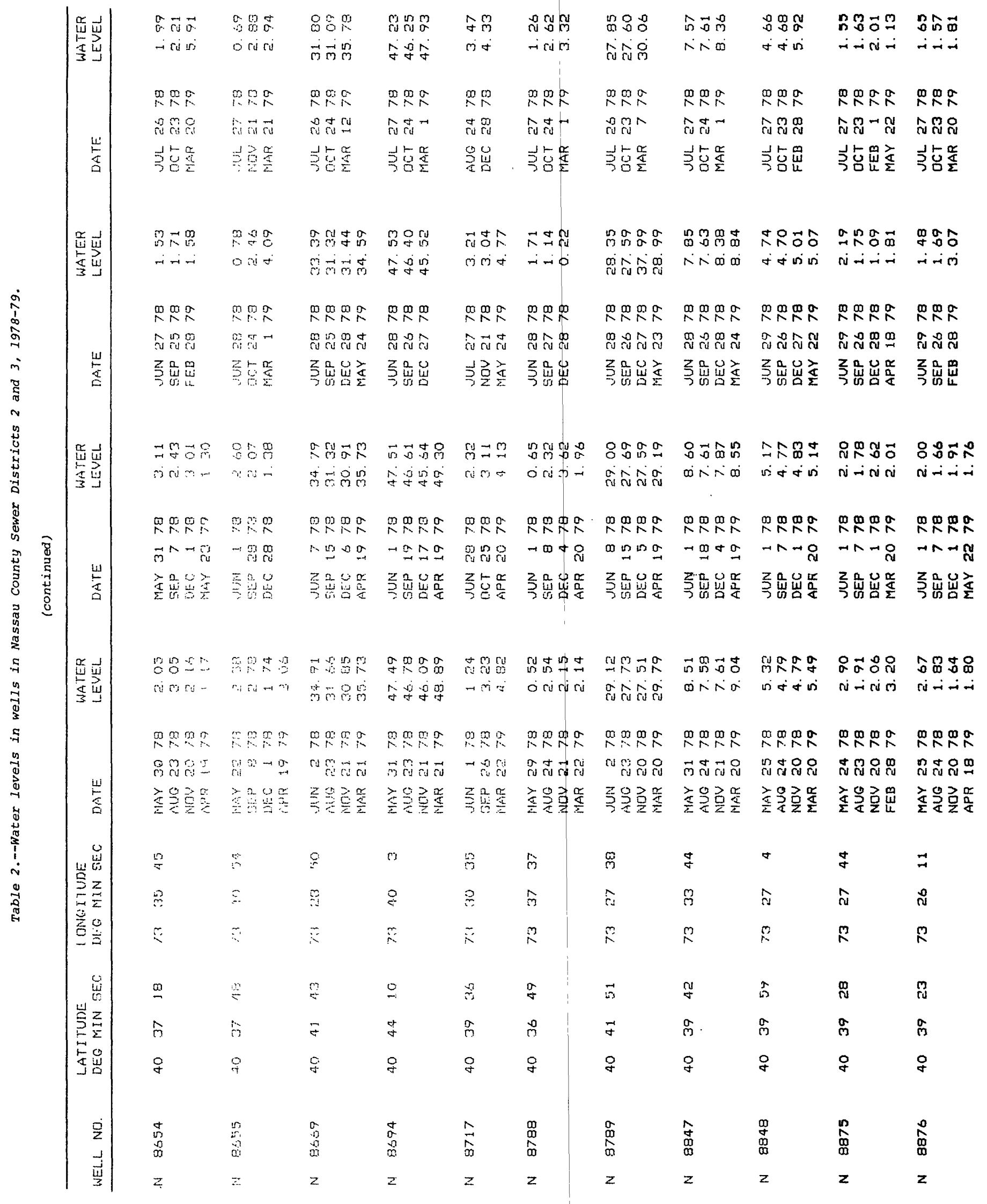




\begin{tabular}{|c|c|c|c|c|c|c|c|c|c|}
\hline \multirow{2}{*}{ 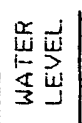 } & $\underset{\infty}{\text { rud }}$ & N品我 & 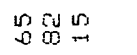 & 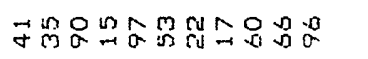 & 80 & $\operatorname{los}_{n}$ & 吕品哭 & 台余票 & 的䞏吉 \\
\hline & bi & ing & og & 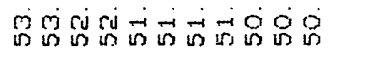 & in is & ㅁo & $\stackrel{n}{n}$ & vidi & gंवें \\
\hline \multirow[b]{3}{*}{ 崖 } & $\underset{r}{r}$ & mog & $\sin \frac{\pi}{\sin }$ & 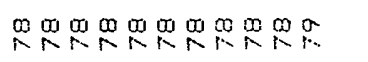 & 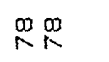 & $m$ & Ren & mag & 象昗 \\
\hline & $\overrightarrow{r u}$ & a sur & 象范禹 & 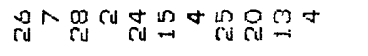 & 跑 & लि ते & ra & 品昆品 & 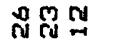 \\
\hline & $\frac{\frac{\alpha}{c}}{\Sigma}$ & 35 & $3 \frac{\alpha}{s}$ & 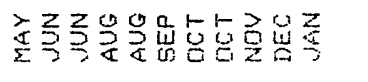 & $\frac{1}{5}$ & 郓 & 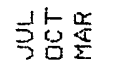 & 引占造 & 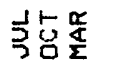 \\
\hline \multirow{2}{*}{ 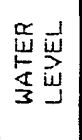 } & $\stackrel{9}{8}$ & 员音品 & 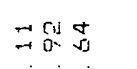 & 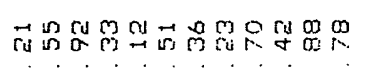 & Nut & 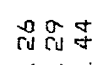 & 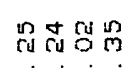 & 옳 & 똣웜 \\
\hline & 亗 & $\sin 6$ & 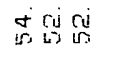 & 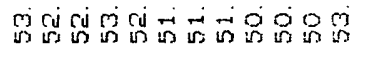 & 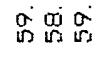 & 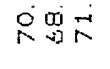 & 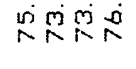 & जिं की & \\
\hline \multirow[b]{3}{*}{$\begin{array}{l}\text { 岁 } \\
+ \\
\end{array}$} & $\stackrel{m}{\wedge}$ & 品只 & $p_{R}^{\infty}$ & 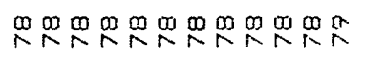 & $\stackrel{\infty}{n} \stackrel{R}{r}$ & pog & megen & meg & 象里只只 \\
\hline & 㫌 & $m_{n \rightarrow \infty}^{n}$ & 唯 & 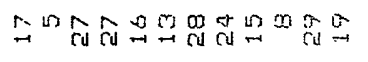 & लिभत लि & लू हैं पे & 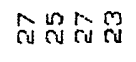 & ลูก & 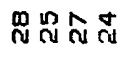 \\
\hline & 岁 & $\sum_{3}^{2} \frac{a}{4}$ & $\varliminf_{3}$ & 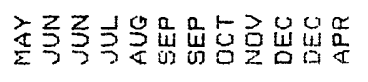 & $\varliminf_{3}^{\frac{a}{w}} \frac{\alpha}{\frac{\alpha}{\alpha}}$ & $3 \frac{1}{3} \frac{7}{2}$ & 高品㟧宾 & 㤩品思 & 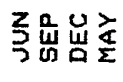 \\
\hline \multirow{2}{*}{ 営 } & $\overrightarrow{0}$ in & 路学 & 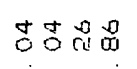 & 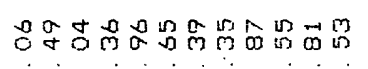 & $2 \%$ & 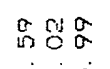 & 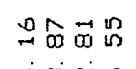 & 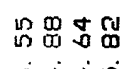 & 员负思合 \\
\hline & 8 & 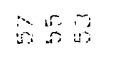 & in & 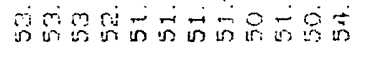 & 究品合 & scos & singe & $\dot{\theta}+\dot{\theta} \dot{\theta}$ & \\
\hline \multirow[b]{3}{*}{$\begin{array}{l}\text { 밈 } \\
\text { 㕿 }\end{array}$} & $\stackrel{R}{\wedge}$ & aga & 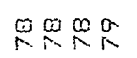 & 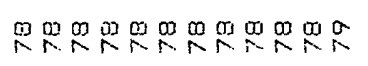 & $\stackrel{p}{M} \stackrel{p}{\wedge}$ & $\stackrel{R}{R}$ & $\underset{\wedge}{\wedge} \operatorname{mog}_{\wedge}^{\infty}$ & 奋RR゚ & 象象果只 \\
\hline & 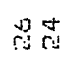 & $\infty\left[\begin{array}{ll}0 \\
\infty\end{array}\right.$ & $\cos \rightarrow 0$ & 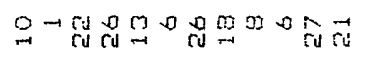 & $\underset{\rightarrow \infty}{\rightarrow \infty}$ & cund & $\neg \underset{\sim}{+\infty} \stackrel{\infty}{\rightarrow}$ & $\vec{M}^{\wedge-M}$ & $-\underset{\sim}{\infty}+\stackrel{\sigma}{-}$ \\
\hline & 它产 & 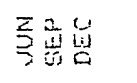 & 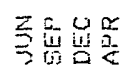 & 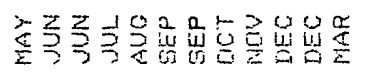 & 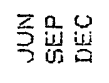 & 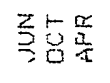 & 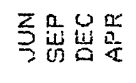 & 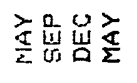 & 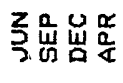 \\
\hline \multirow{2}{*}{ 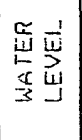 } & $\Rightarrow$ in & 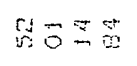 & 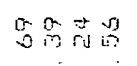 & 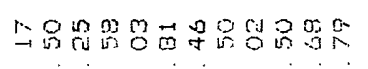 & $\overrightarrow{0} 0$ & 它菏 & 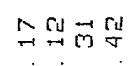 & 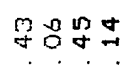 & 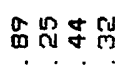 \\
\hline & $\vec{m} \cdot \dot{j}$ & 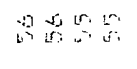 & 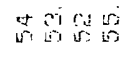 & 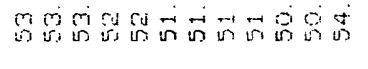 & 可密 & 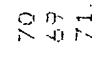 & natis & in $\sin 0$ & 安悉字方 \\
\hline \multirow[b]{3}{*}{ 崩 } & 余是 & 品o & Rog & 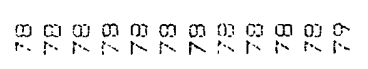 & 我舟品 & $a n a$ & 梁品品 & mpendr & m象负品 \\
\hline & 话 & -482 & - 宓践 & 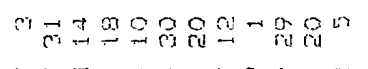 & $\rightarrow$ Cu & $\operatorname{Ban}$ & gmpro & 윰웜 & ज解 Cu \\
\hline & 量竞 & 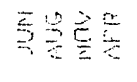 & 总是焉焉 & 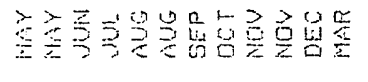 & $3 \frac{0}{3}$ & 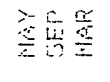 & 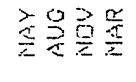 & 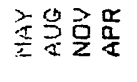 & 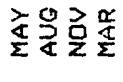 \\
\hline \multirow{3}{*}{ 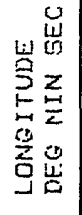 } & $\rightarrow$ & $\vec{s}$ & rid & $\mathrm{s}$ & $\stackrel{M}{F}$ & 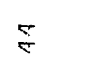 & $\stackrel{\infty}{\square}$ & $m$ & N \\
\hline & ח & $\hat{2}$ & 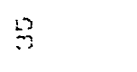 & $\stackrel{2}{m}$ & $\stackrel{m}{p}$ & $\overrightarrow{r i s}$ & $\bar{m}$ & $\vec{n}$ & ले \\
\hline & $\stackrel{M}{R}$ & $\approx$ & $\bar{R}$ & 8 & 2 & 8 & $R^{3}$ & $\stackrel{R}{R}$ & 9 \\
\hline \multirow{3}{*}{ 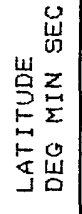 } & $\sigma$ & $\sigma_{i i}$ & $\stackrel{9}{-1}$ & $\sigma$ & $\stackrel{8}{q}$ & $\stackrel{0}{-}$ & $\stackrel{N}{i n}$ & $r_{\text {ru }}^{\text {ru }}$ & y \\
\hline & F & $\stackrel{m}{5}$ & 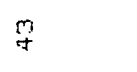 & $\stackrel{3}{q}$ & $\mathscr{q}$ & J্ & $\stackrel{\sigma}{\sigma}$ & $\stackrel{d}{\mathrm{~m}_{1}}$ & $\underset{q}{q}$ \\
\hline & g & ơ & ? & 8 & 9 & $g$ & o & o & \& \\
\hline \multirow{2}{*}{$\begin{array}{l}\frac{0}{z} \\
\frac{1}{\vec{w}}\end{array}$} & 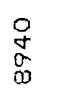 & $\begin{array}{l}m \\
\frac{9}{0} \\
0 \\
0 \\
0\end{array}$ & 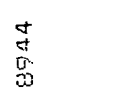 & $\begin{array}{l}0 \\
0 \\
0 \\
0 \\
0 \\
0\end{array}$ & 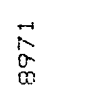 & 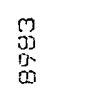 & 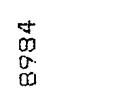 & 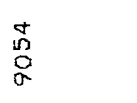 & 垈 \\
\hline & $z$ & $z$ & $I$ & $z$ & $z$ & $z$ & $z$ & $z$ & $z$ \\
\hline
\end{tabular}




\begin{tabular}{|c|c|c|c|c|c|c|c|c|c|c|c|c|}
\hline & \multirow{2}{*}{ 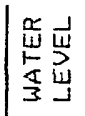 } & 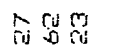 & 空究点 & 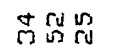 & 号年 & 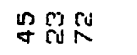 & oㅇ & 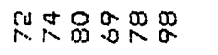 & $\underset{\sigma}{a}$ & $\stackrel{\pi}{\pi}$ & $\vec{o}$ & $\vec{o}$ \\
\hline & & 孚覃品 & 畐别品 & 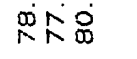 & में & 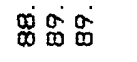 & 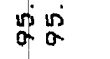 & 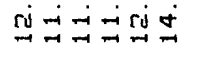 & $\dot{\infty}$ & $\stackrel{\dot{\sigma}}{-}$ & n & $\dot{n}$ \\
\hline & & gag & man & mpr & $\stackrel{m}{\wedge} \stackrel{\mathscr{m}}{\wedge}$ & 虽只 & $\mathbb{R}^{\infty}$ & 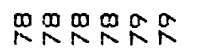 & 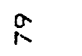 & $\stackrel{\sigma}{\pi}$ & 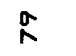 & $\stackrel{\sigma}{R}$ \\
\hline & & तै में & $\mathrm{N}^{3} \mathrm{~S}$ & 品芯等 & ru & 品品め & $\Phi+$ & 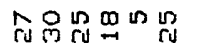 & $\overrightarrow{r u}$ & $\overrightarrow{n d}$ & $\vec{N}$ & $\overrightarrow{\mathrm{N}}$ \\
\hline & $\underset{b}{\underline{G}}$ & $30 \frac{x}{3}$ & 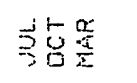 & 引占告 & 号曾 & $5 \frac{\alpha}{5}$ & 品㟧 & 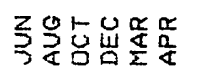 & $\frac{\frac{\alpha}{4}}{2}$ & $\frac{\frac{\alpha}{4}}{\Sigma}$ & $\frac{⿱ 乛}{4}$ & $\frac{\alpha}{2}$ \\
\hline & 学出 & RR̂̃ & 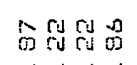 & 柋‡心 & 要喿等 & 可の苟 & 920 & 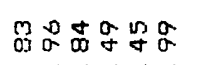 & $\begin{array}{c}\infty \\
n \\
n\end{array}$ & Cu & $\exists$ & 2 \\
\hline & 造出 & $\underset{\sigma}{\sigma} \underset{\sigma}{\sigma} \dot{\sigma}$ & 的的品 & 舟思宫 & 我要品 & 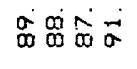 & $\operatorname{dg} \alpha$ & 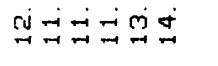 & $r$ & $\stackrel{\sim}{\sim}$ & กี่ & $\vec{n}$ \\
\hline & & m品照尔 & 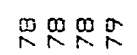 & $\stackrel{m}{\wedge} \stackrel{0}{\wedge}$ & ma & 象里界只 & $\stackrel{m}{\wedge} \stackrel{m}{N}$ & mana & $\stackrel{m}{n}$ & $\stackrel{\infty}{\wedge}$ & $\stackrel{\boldsymbol{\infty}}{\boldsymbol{N}}$ & 界 \\
\hline & & 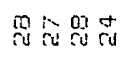 & 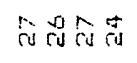 & 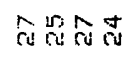 & ำกำ & 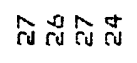 & Mo & 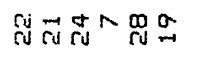 & $\stackrel{\mathbb{N}}{\sim}$ & 罚 & 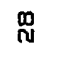 & $\stackrel{\mathfrak{N}}{\mathfrak{N}}$ \\
\hline & 崖 & 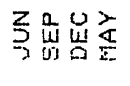 & 强品导高 & 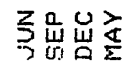 & 尖号妾 & 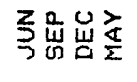 & 等召 & 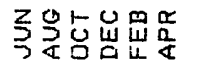 & 㟔 & 㟔 & 岂 & 㟧 \\
\hline & 量 & 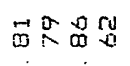 & 分句哀器 & $\overrightarrow{0} \stackrel{0}{*}=$ & 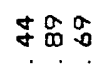 & 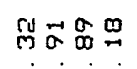 & कीज政 & 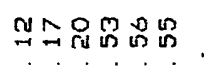 & $\stackrel{n}{N}$ & g $\stackrel{n}{?}$ & R̊ & $\rightarrow 0$ \\
\hline & 它出 & 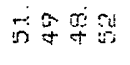 & 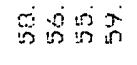 & 究总会 & is & 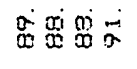 & 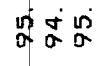 & mad & $\infty \infty$ & $\ddot{-m}$ & ลี่ & $\vec{N} \dot{U}$ \\
\hline & & 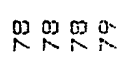 & 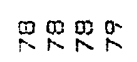 & 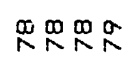 & 果界是 & p & m $\mathbb{R}^{\infty} R$ & ロ & $\stackrel{m a}{n}$ & 界 & 里员 & $\stackrel{p a}{R}$ \\
\hline בู & & $\cos \theta$ & $\neg \ddot{D} \sigma \sigma$ & $n_{\rightarrow} 00$ & 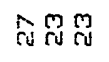 & $\cos _{-1} \sin \theta$ & ลูกู & 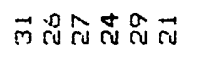 & rug & กี & NN & लू N N \\
\hline $\begin{array}{l}7 \\
\tilde{z} \\
\tilde{g}\end{array}$ & $\frac{\mathrm{L}}{\mathrm{E}}$ & 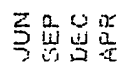 & 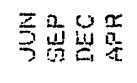 & 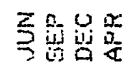 & 妾占品 & 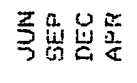 & 可题 & 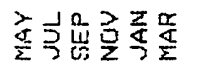 & 官空 & $\frac{\partial}{2}$ & $\frac{\partial}{2} \frac{x}{2}$ & 毫交 \\
\hline & $\frac{1}{3}$ & 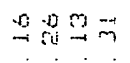 & 9889 & 昌当利只 & $\hat{g}=1$ & 品器品 & ơㅁํㅁ & 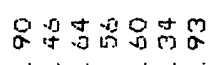 & $8 m$ & $\stackrel{\circ}{\square}$ & 品恕 & in ru \\
\hline & 要步 & 50 & Bog & Rosing & 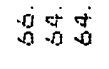 & 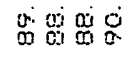 & 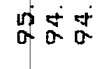 & 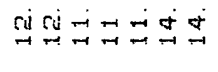 & $\dot{\theta} 0 j$ & $\stackrel{9}{9}$ & ทู่ ชั่ & ลี กุ \\
\hline & & 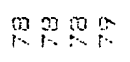 & 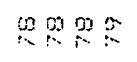 & 血罗品只 & 舟哭是 & 思思 & 舟只只 & 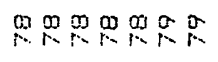 & ma & $\stackrel{R}{\wedge}$ & $\stackrel{\infty}{\wedge}$ & $\frac{m}{R}$ \\
\hline & & मून & $\pi \overrightarrow{r i}$ & 888 คี & คำ & 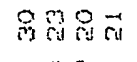 & "यु & 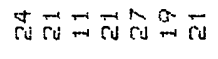 & $\stackrel{4}{a}=$ & rug & $\stackrel{+\pi}{\pi}$ & cas \\
\hline & 容 & 的里 & 证要 & 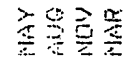 & 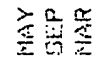 & $\dddot{E}$ & 萧是星 & 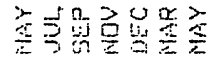 & 骂営 & 占哭 & $5 \frac{\alpha}{0}$ & to \\
\hline & 总 & 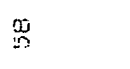 & 9 & ri & 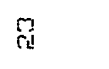 & $\hat{b}$ & 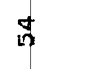 & 9 & $\cong$ & ru & న్ & y \\
\hline & 5 & $\vec{z}$ & 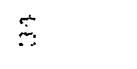 & 8 & 量 & 芯 & 5 & $\stackrel{r u t}{\because}$ & $\stackrel{\infty}{m}$ & $\stackrel{\mathbb{g}}{\circ}$ & 思 & 塄 \\
\hline & 9 & $?$ & $\ddot{n}$ & 8 & $R^{2}$ & $\because$ & $M$ & $R$ & $R^{2}$ & $\stackrel{5}{1}$ & $\stackrel{M}{\wedge}$ & $\sqrt[n]{2}$ \\
\hline & 㟧 & 5 & $\stackrel{5}{*}$ & $\infty$ & $\stackrel{m}{\rightarrow}$ & $\stackrel{\sigma}{=}$ & 9 & $\stackrel{n}{-}$ & 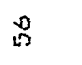 & Y & 品 & f \\
\hline & $1-\Sigma$ & $\stackrel{8}{4}$ & ?ִ & $\underset{v}{i}$ & $\dot{d}$ & $\hat{\varnothing}$ & $\stackrel{0}{q}$ & $\vec{\sigma}$ & $\stackrel{m}{m}$ & $\stackrel{a}{\sigma}$ & 8 & 운 \\
\hline & 我㟧 & 9 & S & 워 & q & 果 & $\stackrel{9}{q}$ & 古 & 웅 & 웅 & 운 & 8 \\
\hline & $\frac{9}{2}$ & 8 & 8 & $\begin{array}{l}\frac{a}{2} \\
0 \\
0\end{array}$ & $\begin{array}{l}\text { w } \\
\text { Oू } \\
\text { o }\end{array}$ & $\begin{array}{l}\text { a } \\
\text { ơ } \\
\text { a }\end{array}$ & $\begin{array}{l}\infty \\
0 \\
0\end{array}$ & $\begin{array}{l}5 \\
\stackrel{9}{0} \\
0 \\
0\end{array}$ & $\begin{array}{l}\vec{a} \\
a \\
a\end{array}$ & $\begin{array}{c}\frac{1}{1} \\
\cdots \\
a\end{array}$ & 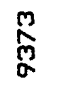 & 苗 \\
\hline & $\frac{\pi}{3}$ & $z$ & $\vec{z}$ & $z$ & $z$ & $z$ & $z$ & $z$ & $z$ & $z$ & $z$ & $z$ \\
\hline
\end{tabular}




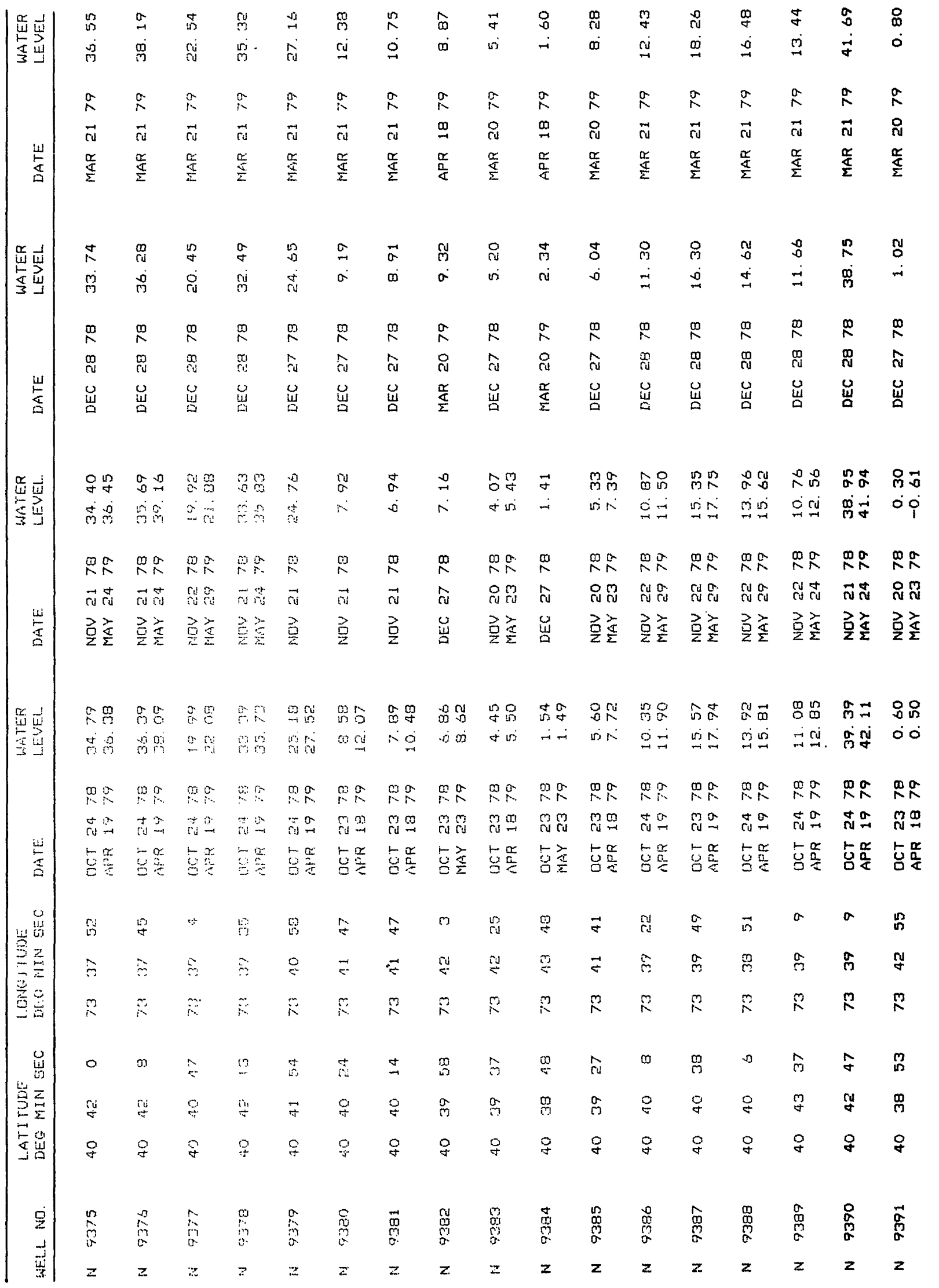




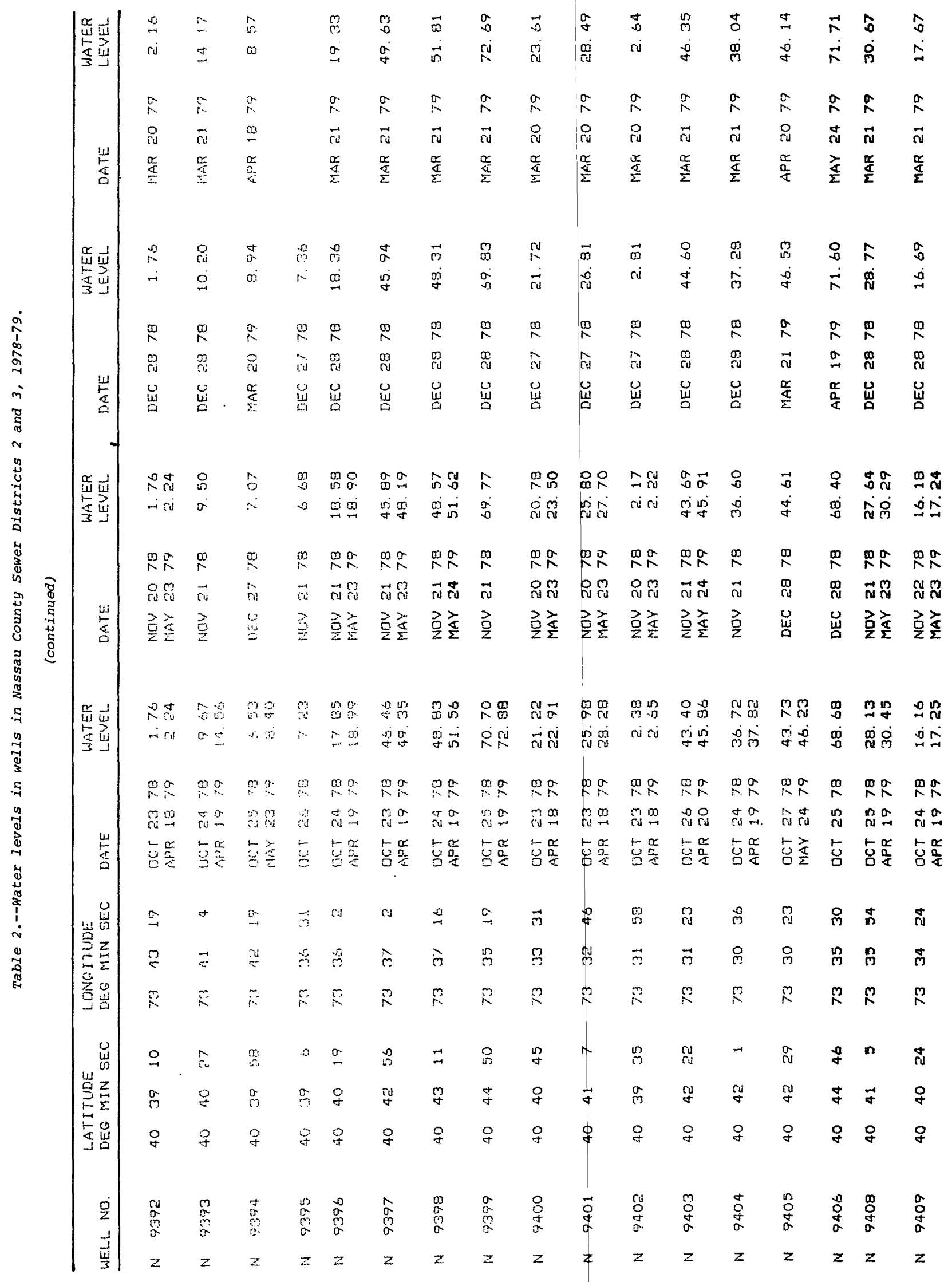




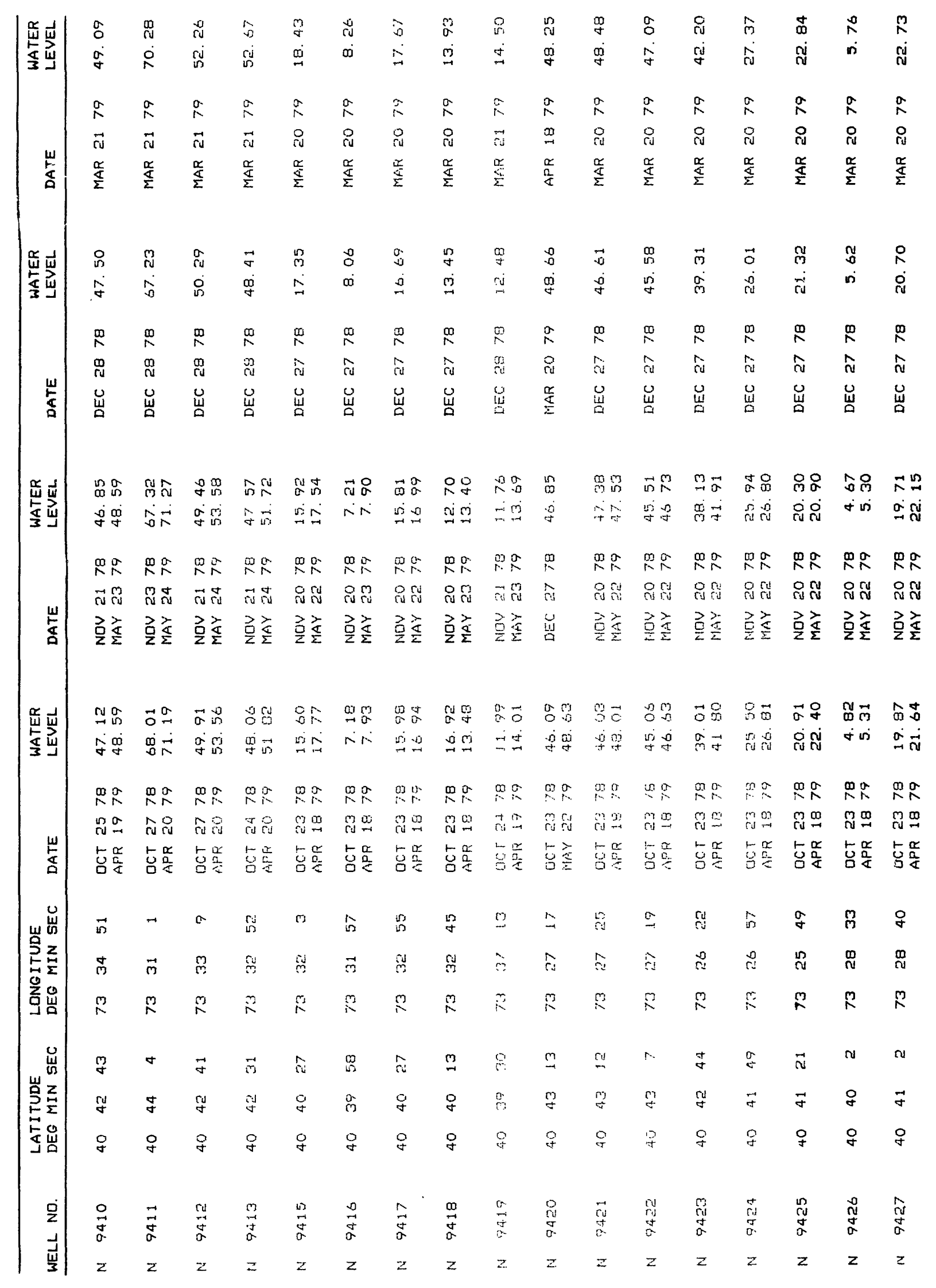




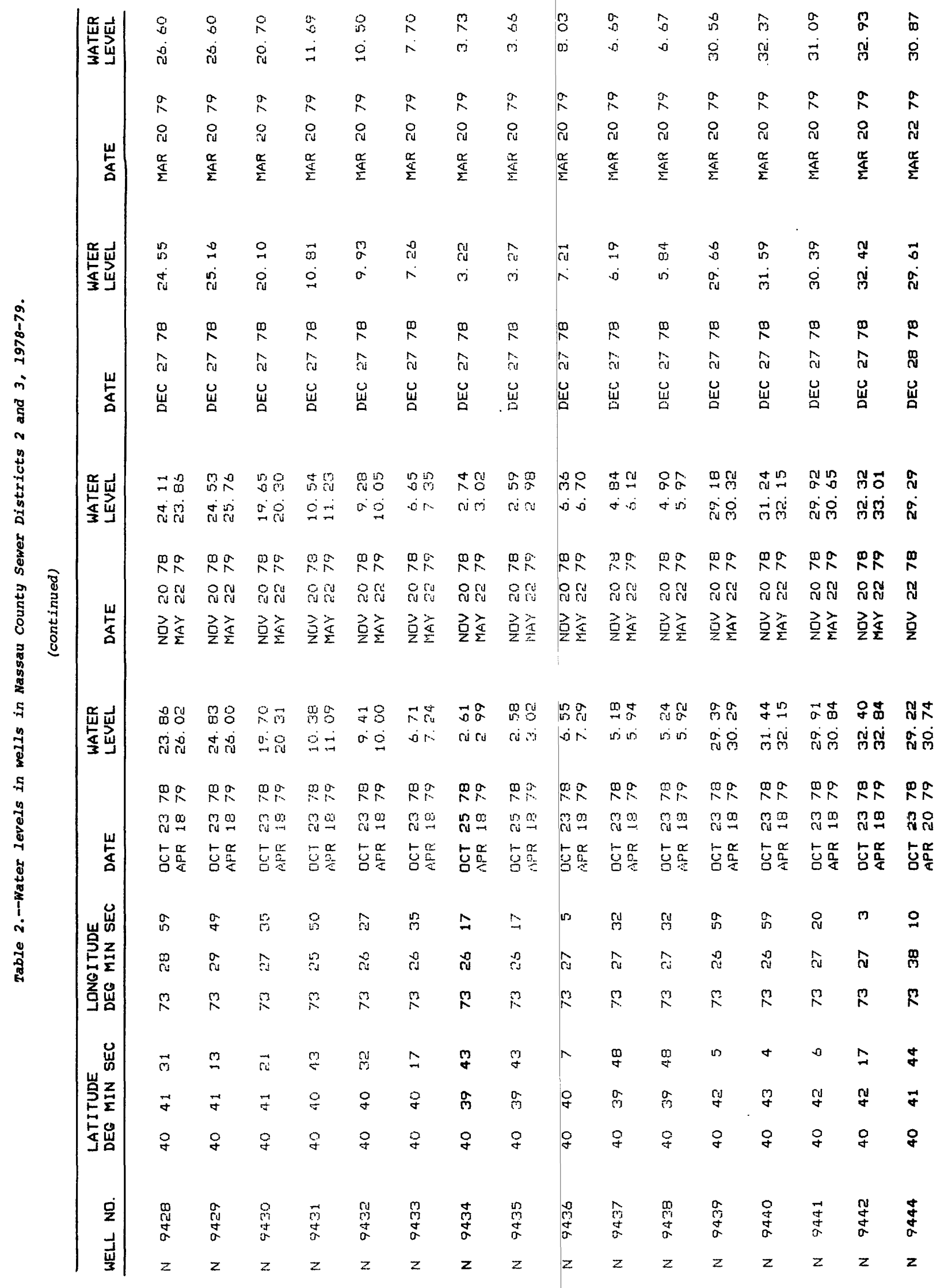

